\title{
$W$-ALGEBRAS, HIGHER RANK FALSE THETA FUNCTIONS, AND QUANTUM DIMENSIONS
}

\author{
KATHRIN BRINGMANN AND ANTUN MILAS
}

\begin{abstract}
Motivated by appearances of Roger's false theta functions in the representation theory of the singlet vertex operator algebra, for each finite-dimensional simple Lie algebra of ADE type, we introduce higher rank false theta functions as characters of atypical modules of certain $W$ algebras and compute asymptotics of irreducible characters which allows us to determine quantum dimensions of the corresponding modules. In the $s \ell_{2}$-case, we recover many results from [10].
\end{abstract}

\section{INTRODUCTION}

It is well known (due to Frenkel-Kac and Segal) that any simply-laced affine Lie algebra $\widehat{\mathfrak{g}}$ admits a homogeneous realization in terms of vertex operators acting on a direct sum of infinitely many Fock spaces. More precisely, for every coset $\lambda+Q \in Q^{0} / Q$ ( $Q$ the root and $Q^{0}$ the weight lattice) there is an irreducible highest weight $\widehat{\mathfrak{g}}$-module $V_{Q+\lambda}$. This construction can be reformulated (due to Borcherds [8]; see also [23]) in the language of vertex operator algebras. From this point of view, the module $V_{Q}$ has a vertex operator algebra structure (lattice vertex algebra) while $V_{Q+\lambda}$ is an irreducible $V_{Q}$-module [29]. Under the standard grading, characters of these modules are known and given by

$$
\operatorname{ch}\left[V_{Q+\lambda}\right](\tau)=\frac{\sum_{\alpha \in Q+\lambda} q^{\frac{\|\alpha\|^{2}}{2}}}{\eta(\tau)^{\operatorname{rank}(Q)}},
$$

where $\|\alpha\|^{2}:=(\alpha, \alpha)$ and $\eta(\tau):=q^{\frac{1}{24}} \prod_{n \geq 1}\left(1-q^{n}\right)$ is Dedekind's $\eta$-function $\left(q:=e^{2 \pi i \tau}\right.$ throughout $)$, a modular form of weight $1 / 2$. Modular forms have appeared in abundance in the representation theory of rational vertex operator algebras [29], especially in the theory surrounding the moonshine phenomena [23].

In the last ten years, a lot of research has been devoted to understanding the so called logarithmic conformal field theories and related irrational vertex algebras $[2,4,18,19,20,24,30$, 32], etc. In these theories, modularity either holds with some modifications, as seen in $C_{2}$-cofinite vertex operator algebras (VOAs), or is completely absent as is the case of non $C_{2}$-cofinite VOAs. An interesting feature of irrational $C_{2}$-cofinite vertex algebras is the lack of an affine Lie algebra action. One reason for this comes from the fact that all known examples of such vertex algebras appear as proper subalgebras of vertex algebras associated to dilated root lattices $\sqrt{p} Q$, with $p \in \mathbb{N}_{\geq 2}$ (here $p$ is not necessarily prime!). Although these rootless lattices rarely appear in Lie theory, they do have relevance in vertex algebra theory.

In many ways this paper is a continuation of [10] (see also $[11,14,30]$ ) where the authors thoroughly studied properties of certain false theta functions coming from characters of modules of the singlet vertex operator algebra. The starting point in this line of work was an observation

2010 Mathematics Subject Classification. 17B69, 14K25.

Key words and phrases. characters, false theta functions, Jacobi forms, modular forms, vertex algebras. 
$[10,11]$ that the classical Roger's false function

$$
\sum_{n \in \mathbb{Z}} \operatorname{sgn}(n) q^{p\left(n+\frac{j}{2 p}\right)^{2}}=\sum_{n=0}^{\infty} q^{p\left(n+\frac{j}{2 p}\right)^{2}}-\sum_{n=0}^{\infty} q^{p\left(n+\frac{n-j}{2 p}\right)^{2}}
$$

and other related false theta functions show up as "numerators" of characters of the $(1, p)$-singlet $W$-algebra (see [1]).

In the theory of modular forms, false theta series have occurred in many important works, including $[6,28,34,36]$. These are not modular but when passing to the lower half-plane radially one obtains mock modular forms which are holomorphic parts of so-called harmonic Maass forms (non-holomorphic modular forms annihilated by the Laplace operator). In particular, this yields an analytic function in the upper half-plane which drips into the lower half-plane.

It is already known that the $(1, p)$-singlet algebra is related in a non-trivial way to the Lie algebra $s \ell_{2}$, so this raises the question of whether functions like (1.1) can be defined for other root systems such that they also give rise to characters of modules. As we previously announced in [30], with full details given here, this is indeed possible, at least in the simply-laced case.

To explain our construction, we first consider a sequence of embeddings of vertex algebras:

$$
\mathcal{W}_{c_{p}}(\mathfrak{g}) \hookrightarrow W^{0}(p)_{Q} \hookrightarrow W(p)_{Q} \hookrightarrow V_{\sqrt{p} Q},
$$

where $\mathfrak{g}$ is the corresponding simple Lie algebra, $\mathcal{W}_{c_{p}}(\mathfrak{g})$ is the affine $W$-algebra associated to $\mathfrak{g}$ $[7,15]$, and $W^{0}(p)_{Q}$ and $W(p)_{Q}$ are certain vertex algebras defined below. In the special case of $\mathfrak{g}=\mathrm{s} \ell_{2}$, we recover a more familiar embedding of vertex algebras studied for examples in $[2,10,11]$ :

$$
L\left(c_{p, 1}, 0\right) \hookrightarrow W(2,2 p-1) \hookrightarrow W(p) \hookrightarrow V_{\sqrt{2 p} \mathbb{Z}},
$$

where $W(2,2 p-1)$ is the $(1, p)$-singlet vertex algebra [1].

It turns out that the most interesting $q$-series come from considerations of characters of $W^{0}(p)_{Q^{-}}$ modules. The vertex algebra $W^{0}(p)_{Q}$ is not $C_{2}$-cofinite and as such admits characters of modules with peculiar properties. In a special case of the vertex algebra $W^{0}(p)_{Q}$, we get the following character formula

$$
\sum_{\alpha \in Q \cap P^{+}} \operatorname{dim}\left(V(\alpha)_{0}\right) \sum_{w \in W}(-1)^{\ell(w)} \frac{q^{\frac{1}{2}\left\|\sqrt{p} w(\alpha+\rho)-\frac{1}{\sqrt{p}} \rho\right\|^{2}}}{\eta(\tau)^{\operatorname{rank}(Q)}},
$$

with $V(\alpha)_{0}$ the zero weight space of the irreducible highest weight $\mathfrak{g}$-module $V(\alpha), W$ is the Weyl group, $\ell(w)$ is the length of $w$, and $P^{+}$denotes the set of dominant integral weights. The expression (1.2), after eliminating the $\eta$-factor, should be viewed as a higher rank generalization of the classical false theta functions. Indeed, the sign factor in (1.1) can be understood as $(-1)^{\ell(w)}$, where $w$ is the non-unit Weyl group element of $s \ell_{2}$. As far as we know, $q$-series like (1.2) have not been studied in the literature apart from the rank one case. We believe that they are closely related to certain limits of colored Jones polynomials of alternating knots colored by representations of $\mathfrak{g}$, studied in [25]. Connection between the $(1, p)$ singlet characters and certain torus knots colored by $\mathfrak{s l}_{2}$ representations was already established in [10]. Another, more conceptual, contact between this line of work and quantum knot (and link) invariants is expected to be obtained by establishing equivalences of categories of $W^{0}(p)_{Q}$-modules and modules for "unrolled" quantum groups at root of unity. For the $(1, p)$ singlet algebra, this equivalence was recently studied in [13].

The aim of this paper is to study properties of characters of $W(p)_{Q}$ initiated in [18] and especially characters of $W^{0}(p)_{Q^{-}}$-modules such as (1.2). We briefly outline the paper and indicate the main results. We introduce vertex operator algebras $W(p)_{Q}$ and $W^{0}(p)_{Q}$ in Section 2, following mostly [4, $18,30]$, although similar constructions with screening operators can be traced to affine $W$-algebras [22]. In Section 3, we recall modular transformation properties of higher rank theta functions coming from lattices, while, in Section 4 , we discuss characters of certain $W(p)_{Q}$-modules following 
closely the proposal in [18]. Our main result is Theorem 4.1 below, proving finite-dimensionality of the $\mathrm{SL}_{2}(\mathbb{Z})$-closure of the space of $W(p)_{Q^{-}}$characters. We also work out in full detail modular transformation properties of $\operatorname{ch}\left[W(p)_{A_{2}}\right](\tau)$ (see Section 4.3). Section 5 is primarily concerned with

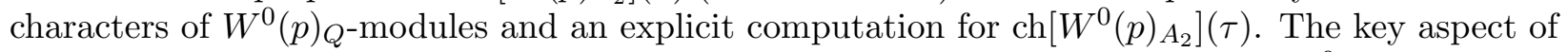
this paper is in Section 6, where we study asymptotic expansions of characters of $W^{0}(p)_{Q}$-modules. The main result here is Theorem 6.2 , which can be restated as follows.

Theorem 1.1. For $\beta \in L^{0}$, as $t \rightarrow 0^{+}$,

$$
\eta(i t)^{\operatorname{dim}(Q)} \operatorname{ch}\left[W^{0}(p, \beta)_{Q}\right](i t) \sim \frac{\operatorname{dim}(V(-\sqrt{p} \bar{\lambda}))}{p^{\left|\Delta_{+}\right|}}+O(\sqrt{t}) .
$$

where $\bar{\lambda}$ (depending on $\beta$ ) is defined in Section 5.1 .

Although our method for computing asymptotic expansion of $W^{0}(p)_{Q}$ is very general, for brevity in Section 7 we only compute the full expansion of $\operatorname{ch}\left[W^{0}(p)_{A_{2}}\right](\tau)$. We obtain

Theorem 1.2. Asymptotically, as $t \rightarrow 0^{+}$, we have with $p \in \mathbb{N}_{\geq 2}$,

$$
\eta(i t)^{2} \operatorname{ch}\left[W^{0}(p)_{A_{2}}\right](i t) \sim \sum_{N \geq 0} \alpha(N)(-2 \pi p t)^{N}
$$

where $\alpha(N)$ is defined in (7.6) below.

Remark 1. For $Q=A_{1}$, in [10], the authors obtained an asymptotic expansion of characters. This asymptotic expansion was then related to Eichler integrals which have a nice (mock transformation) behavior. We plan to investigate in future research whether the formulas in Theorem 1.2 can be used to find companions of the characters in the lower half-plane which is (mock) modular.

For regular vertex operator algebras (or more generally, modular tensor categories) a quantum dimension defines a homomorphism from the Grothendick ring of the category of modules to $\mathbb{C}$. If we denote by $M_{j}, 0 \leq j \leq k$, all inequivalent irreducible $V$-modules, where we pick $V=M_{0}$, the quantum dimension of $M_{j}$ is given by

$$
\operatorname{qdim}\left(M_{j}\right)=\frac{S_{j, 0}}{S_{0,0}}
$$

where $S_{j, k}$ are the entries of the $S$-matrix. It is expected that this quantity plays an important role for irrational vertex algebras as well. Thus, in Section 8, based on results of Section 6, we compute analytic quantum dimensions of certain $W^{0}(p)_{Q}$-modules. This quantum dimension is defined purely analytically as the limit

$$
\operatorname{qdim}(M):=\lim _{t \rightarrow 0+} \frac{\operatorname{ch}[M](i t)}{\operatorname{ch}[V](i t)} .
$$

We stress that for regular vertex operator algebra these two definitions agree, at least if lowest conformal weights of $M_{j}, j \neq 0$, are positive. By using the last definition, we show in Section 8 that

Corollary 1.3. For $\beta \in L^{0}$, whose coset representative in $L^{0} / L$ is $\lambda$, we have

$$
\operatorname{qdim}\left(W^{0}(p, \beta)_{Q}\right)=\operatorname{dim}_{\mathbb{C}} V(-\sqrt{p} \bar{\lambda})
$$

where $\bar{\lambda}$ is defined in (4.1). 


\section{ACKNOWLEDGMENTS}

The research of the first author (K.B.) is supported by the Alfried Krupp Prize for Young University Teachers of the Krupp foundation and the research leading to these results recieves funding from the European Research Council under the European Union's Seventh Framework Programme (FP/2007-2013) / ERC Grant agreement n. 335220 - AQSER. The second author (A.M) was partially supported on a Simons Foundation grant and support from the Max Planck Institute for Mathematics, Bonn. The authors thank Matt Krauel for helpful comments on an earlier version of this paper and Karl Mahlburg, Mike Woodbury, and Don Zagier for enlightening conversations. We also thank Sander Zwegers for very useful comments in connection to Theorem 4.1. Finally, we thank the referee for constructive comments.

\section{Vertex operator algebras, $W(p)_{Q}$ And $W^{0}(p)_{Q}$}

Let $(Q,(\cdot, \cdot))$ be a rank $n$ root lattice of ADE type, and $Q^{0}=P$ its dual (weight) lattice. We are primarily concerned with dilated root lattices $L=\sqrt{p} Q$ and their duals $L^{0}$, where $p \in \mathbb{N}_{\geq 2}$. Let $\alpha_{j}, 1 \leq j \leq n$, denote the simple roots of $Q$, and $\Delta_{+}$and $\Delta_{-}$the set of positive and negative roots, respectively. We have $\left\|\alpha_{j}\right\|^{2}=\left(\alpha_{j}, \alpha_{j}\right)=2$ for all $j$.

We equip $V_{L}$ with a vertex algebra structure by choosing an appropriate 2-cocycle as in the case of $V_{Q}[4,29,31]$. For the conformal vector we choose $[2,18,30,31]$

$$
\omega=\omega_{0}+\frac{p-1}{2 \sqrt{p}} \sum_{\alpha \in \Delta_{+}} \alpha(-2) \mathbf{1},
$$

where $\omega_{0}$ denotes the canonical (quadratic) Virasoro generator [29]. Then $V_{L}$ is a conformal vertex algebra of central charge

$$
\operatorname{rank}(Q)+12(\rho, \rho)\left(2-p-\frac{1}{p}\right)
$$

where $\rho$ denotes the half-sum of positive roots. Next, we consider screening operators $e_{0}^{\sqrt{p} \alpha_{j}}, e_{0}^{-\frac{\alpha_{k}}{\sqrt{p}}}$ $(1 \leq j, k \leq n)$ acting among $V_{L}$ and certain $V_{L}$-modules. It is known that for every $j$ and $k$ the operators $e_{0}^{\sqrt{p} \alpha_{j}}$ and $e_{0}^{-\alpha_{k} / \sqrt{p}}$ commute with each other, and they both commute with the Virasoro algebra $[4,18,31]$.

The connection to affine $W$-algebras is achieved via screening operators. Recall that there are two methods for defining universal affine $W$-algebras. The affine $W$-algebra associated to $\widehat{\mathfrak{g}}$ at level $k \neq-h^{\vee}$, denoted by $\mathcal{W}_{k}(\mathfrak{g})$, is usually defined as the cohomology group obtained via a quantized BRST complex for the Drinfeld-Sokolov hamiltonian reduction [22]. Cohomology of this complex is nontrivial only in degree zero [22]. Moreover, it is known that $\mathcal{W}_{k}(\mathfrak{g})$ is freely generated by primary fields as a vertex algebra (thus the name $W$-algebra), in the sense that it is strongly freely generated by $\operatorname{rank}(\mathfrak{g})$ primary fields, not counting the conformal vector. On the other hand, an important result by B. Feigin and E. Frenkel [22] says that for $k$ generic and $\mathfrak{g}$ simply-laced, there is an alternative description of $\mathcal{W}_{k}(\mathfrak{g})$ in terms of free fields. We use $F_{0}$ to denote the rank $n$ vacuum Fock space, which is also a vertex algebra, and by $F_{\lambda}$ Fock modules parametrized by $\mathfrak{h}$. Let $\nu=k+h^{\vee}$, where $k$ is generic and consider the following operators [22]:

$$
e_{0}^{-\frac{\alpha_{j}}{\sqrt{\nu}}}: F_{0} \longrightarrow F_{-\frac{\alpha_{j}}{\sqrt{\nu}}} \quad(1 \leq j \leq n)
$$

Then

$$
\mathcal{W}_{\nu}(\mathfrak{g})=\bigcap_{j=1}^{n} \operatorname{Ker}_{F_{0}}\left(e_{0}^{-\frac{\alpha_{j}}{\sqrt{\nu}}}\right)
$$


Moreover, we also have the following important duality

$$
\mathcal{W}_{\nu}(\mathfrak{g})=\bigcap_{j=1}^{n} \operatorname{Ker}_{F_{0}}\left(e_{0}^{\sqrt{\nu} \alpha_{j}}\right) .
$$

For non-generic values, screening operators can still be used to define vertex algebras which we now describe. For instance, $p=k+h^{\vee} \in \mathbb{N}_{\geq 2}$ is known to be non-generic. So we let

$$
W^{0}(p)_{Q}:=\bigcap_{j=1}^{n} \operatorname{Ker}_{F_{0}} e_{0}^{-\frac{\alpha_{j}}{\sqrt{p}}},
$$

a vertex operator algebra containing $\mathcal{W}_{p}(\mathfrak{g})$ as a proper subalgebra $[4,12,18]$. In particular, for $Q=A_{1}, W(p)_{Q}$ is precisely the $(1, p)$-singlet algebra discussed in [10,14]. Because of the integrality condition, the previous algebra can be maximally extended within the lattice vertex algebra leading to $[2,12,18,32]$

$$
W(p)_{Q}:=\bigcap_{j=1}^{n} \operatorname{Ker}_{V_{L}} e_{0}^{-\frac{\alpha_{j}}{\sqrt{p}}} .
$$

Again, if we let $Q=A_{1}$, this is the well-known triplet vertex operator algebra $W(p)$. Similarly, we can construct irreducible $W(p)_{Q}$-modules. The following conjecture was mentioned in $[4,18]$.

Conjecture 1. The vertex operator algebra $W(p)_{Q}$ is $C_{2}$-cofinite.

Remark 2. Due to the complicated embedding structure of higher Feigin-Fuchs modules, there are essentially no results on the representation theory of $W^{0}(p)_{Q}$ and of $W(p)_{Q}$, for $\operatorname{rank}(Q) \geq 2$. Still, in [18], the authors proposed an interesting geometric method for studying $W(p)_{Q^{-}}$-modules and their characters. In particular, it is expected that all irreducible representations of $W(p)_{Q}$ can be understood as subquotients of $V_{\sqrt{p} Q}$-modules $[4,18,30]$. Since we are only interested in properties of characters of modules, from now on, we assume that the modules in [18] provide a complete list of irreducible $W(p)_{Q}$-modules and characters (this is yet to be proven by using methods of vertex algebras as in [2]). We also conjecture that all atypical irreducible representations of $W^{0}(p)_{Q}$ appear in the decomposition of irreducible $W(p)_{Q}$-modules. Typical representations are modules isomorphic to higher rank Fock spaces.

\section{Theta functions Associated to LAtTiCES}

This short section deals with higher rank theta functions. All results presented here are already contained in the literature, but we recall them for the reader's convenience.

For $\tau \in \mathbb{H}, M \in \operatorname{Pos}\left(n ; \mathbb{R}\right.$ ) (positive definite matrices with real entries) and $a, b \in \mathbb{C}^{n}$, we define

$$
\Theta_{a, b}(\tau ; M):=\sum_{r \in \mathbb{Z}^{n}} e^{\pi i \tau(r+a)^{T} M(r+a)+2 \pi i(r+a)^{T} b} .
$$

Then

$$
\Theta_{-b, a}\left(-\frac{1}{\tau} ; M^{-1}\right)=(-i \tau)^{\frac{n}{2}} \sqrt{\operatorname{det}(M)} e^{-2 \pi i a^{T} b} \Theta_{a, b}(\tau ; M) .
$$

We also present essentially the same result using different notation. Let $(L,()$,$) be a positive$ definite integral lattice and $L^{\circ}$ denotes its dual. For $g \in \mathbb{Q} \otimes L$, define

$$
\vartheta_{L, g}(u ; \tau):=\sum_{\alpha \in L} e^{\pi i \tau\|\alpha+g\|^{2}+2 \pi i(\alpha+g, u)}
$$

where $u \in \mathbb{C} \otimes L \cong \mathbb{C}^{\operatorname{rank}(L)}$ and $\|\gamma\|^{2}:=(\gamma, \gamma)$. Denoting by $A$ the Gram matrix of $L$, we have

$$
\Theta_{g, u}(\tau ; A)=\vartheta_{L, g}(u ; \tau) \text {, }
$$


where $n:=\operatorname{rank}(L)$. The next result is well-known (see e.g. [26]).

Theorem 3.1. For $[g]=g+L \in L^{\circ} / L$, we have

$$
\vartheta_{L, g}\left(u ;-\frac{1}{\tau}\right)=\frac{(-i \tau)^{\frac{m}{2}}}{\sqrt{\operatorname{det}(A)}} e^{\pi i u^{2}} \sum_{k=0}^{m-1} \zeta_{n}^{k} \vartheta_{L_{g}, k r}(\tau u ; \tau),
$$

where $m:=o([g])$ (order of $[g]$ in $\left.L^{\circ} / L\right), r$ satisfies $(r, g) \equiv \frac{1}{m}(\bmod 1)$ and $L_{g}:=\{\alpha \in L:(\alpha, g) \in$ $\mathbb{Z}\}$, an intermediate lattice of $L$ and $L^{0}$.

\section{Characters of $W(p)_{Q}$-MOdules}

4.1. The Weyl character formula. As in Section 2, let $L=\sqrt{p} Q$, where $Q$ is an ADE root lattice and $\rho$ is the half-sum of positive roots. Also, denote by $W$ the Weyl group. Set

$$
\Delta:=\prod_{\alpha \in \Delta_{-}}\left(1-e^{\alpha}\right)
$$

the (formal) Weyl denominator. We can also view $\Delta$ as a complex valued function. We introduce complex variables $z_{j}$ and let $\mathbf{z}^{\alpha}=z_{1}^{\left(\omega_{1}, \alpha\right)} \cdots z_{n}^{\left(\omega_{n}, \alpha\right)}$, where $n=\operatorname{rank}(Q)$. We also let $z_{j}=e^{2 \pi i u_{j}}, u_{j} \in \mathbb{C}$. Then, for $z=\left(z_{1}, \ldots, z_{n}\right)$, we define

$$
\Delta(z):=\prod_{\alpha \in \Delta_{-}}\left(1-\mathbf{z}^{\alpha}\right)
$$

This function should not to be confused with the modular discriminant!

Let $w \in W$ act on $\mathbb{C}\left[\mathfrak{h}^{*}\right]$, where $\mathfrak{h}^{*}$ is the dual of the Cartan subalgebra, according to $w \cdot e^{\gamma}=e^{w(\gamma)}$ and similarly for $w$ acting on $\mathbf{z}^{\gamma}$. Then we have the well known formula

$$
w\left(\prod_{\alpha \in \Delta_{-}}\left(1-e^{\alpha}\right)\right)=e^{\rho-w(\rho)}(-1)^{\ell(w)} \prod_{\alpha \in \Delta_{-}}\left(1-e^{\alpha}\right) .
$$

For $\lambda \in P^{+}$(dominant integral weight) we denote by $\chi_{\lambda}$ the character of the highest weight module. Recall also the Weyl Character Formula:

$$
\chi_{\lambda}(z)=\sum_{w \in W}(-1)^{\ell(w)} \frac{\mathbf{z}^{w(\lambda+\rho)-\rho}}{\Delta(z)} .
$$

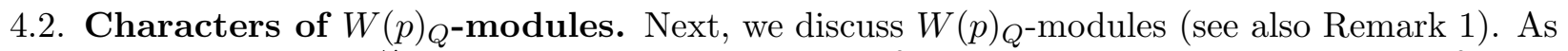
in [18], we choose $\lambda_{j}=\frac{\omega_{j}}{\sqrt{p}}, j=1, \ldots, n$, to be a basis of $L^{0}$ and we also fix representative of $Q^{0} / Q$ to be $0, \omega_{1}, \ldots, \omega_{n}$ for type $A_{n}$, where $\omega_{j}$ are the fundamental weights. For $D_{n}$, we take $0, \omega_{1}, \omega_{n-1}, \omega_{n}$; for $E_{6}$ we choose $0, \omega_{1}$ and $\omega_{3}$; for $E_{7}$ we take 0 and $\omega_{2}$, and for $E_{8}$ we choose only 0.

Again, following [18], each coset $L^{0} / L$ has a unique representative $\lambda$ of the form

$$
\lambda=\sqrt{p} \widehat{\lambda}+\sum_{j=1}^{n}\left(1-s_{j}\right) \lambda_{j}
$$

where $\hat{\lambda}$ is the representative of $Q^{0} / Q$ fixed earlier, and $\bar{\lambda}=\sum_{j=1}^{n}\left(1-s_{j}\right) \lambda_{j}$ such that $s_{j} \in$ $\{1,2, \ldots, p\}$. From now on, elements $\lambda \in L^{0} / L$ are identified with a unique representative of cosets fixed above. 
Example 1. For $Q=A_{2}$, there are $3 p^{2}$ cosets of $L^{0} / L$. For each $\widehat{\lambda} \in\left\{0, \omega_{1}, \omega_{2}\right\}$ there are $p^{2}$ choices of $\bar{\lambda}$, namely:

$$
\bar{\lambda}=\left(1-s_{1}\right) \frac{\omega_{1}}{\sqrt{p}}+\left(1-s_{2}\right) \frac{\omega_{2}}{\sqrt{p}}, \quad s_{1}, s_{2} \in\{1, \ldots, p\} .
$$

To each coset representative $\lambda \in L^{0} / L$ as above, we now associate an irreducible $W(p)_{Q^{-m o d u l e}}$ denoted by $W(p, \lambda)_{Q}$; there are $p^{n}\left|Q^{0} / Q\right|$ such modules in total. As mentioned in the introduction, we omit detailed discussion about modules here. Instead we only focus on their characters (also known as graded dimensions) and refer the reader to [18]. As it is customary in vertex algebra theory, the character of a module $M$ is defined as

$$
\operatorname{ch}[M](\tau):=\operatorname{tr}_{M} q^{L(0)-\frac{c}{24}},
$$

where $c$ is the central charge. But for $W(p)_{Q^{-}}$-modules, it is also convenient to have on disposal complex variables $z_{j}$ (and $u_{j}$ ). These extra variable should come from yet to be constructed action of the simple Lie algebra $\mathfrak{g}$ on $W(p)_{Q}$ (for $\mathrm{s} \ell_{2}$ this action was constructed in [5]). The resulting expression is called a full character. Then we have the following formulas for full characters of atypical irreducible $W(p)_{Q}$-modules proposed in [18]:

$$
\begin{aligned}
\operatorname{ch}\left[W(p, \lambda)_{Q}\right](\tau, z) & =\frac{1}{\eta(\tau)^{\operatorname{rank}(Q)}} \sum_{w \in W} \sum_{\alpha \in Q}(-1)^{\ell(w)} \frac{q^{\frac{1}{2}\left\|\sqrt{p} \alpha+\lambda+\left(\sqrt{p}-\frac{1}{\sqrt{p}}\right) \rho\right\|^{2}} \mathbf{z}^{w(\alpha+\widehat{\lambda})}}{w(\Delta(z))} \\
& =\frac{1}{\eta(\tau)^{\operatorname{rank}(Q)}} \sum_{\alpha \in Q} q^{\frac{1}{2}\left\|\sqrt{p}(\alpha+\rho+\widehat{\lambda})+\bar{\lambda}-\frac{1}{\sqrt{p}} \rho\right\|^{2}}\left(\sum_{w \in W}(-1)^{\ell(w)} \frac{\mathbf{z}^{w(\alpha+\rho+\widehat{\lambda})-\rho}}{\Delta(z)}\right) .
\end{aligned}
$$

Observe that the expression in the parentheses of (4.2) does not necessarily equal the Weyl character unless $\alpha+\widehat{\lambda} \in P^{+}$; rather it can be expressed as the Weyl character of different highest weight, up to a sign. As we shall see momentarily, this formula is very useful for determining modular properties of $\operatorname{ch}\left[W(p, \lambda)_{Q}\right](\tau, z)$ after $z \rightarrow 1$ (i.e., $\left.u \rightarrow 0\right)$.

Next, we rewrite (4.2) by adjusting the Weyl sum (cf. [18]). Observe that we get a non-zero contribution in the sum only if $\alpha+\rho+\widehat{\lambda}$ is in the interior of a Weyl chamber. For such $\alpha+\rho+\widehat{\lambda}$, there is $w \in W$ such that $w(\alpha+\rho+\hat{\lambda})$ is inside the fundamental Weyl chamber. Thus we can replace the summation over the root lattice as a summation over the $Q \cap P^{+}$and an extra summation over the Weyl group. We use this and (4.2) to get

$$
\begin{aligned}
\operatorname{ch}\left[W(p, \lambda)_{Q}\right](\tau, z) & =\frac{1}{\eta(\tau)^{\operatorname{rank}(Q)}} \sum_{w \in W} \sum_{\alpha \in Q}(-1)^{\ell(w)} \frac{q^{\frac{1}{2}\left\|\sqrt{p}(\alpha+\rho+\widehat{\lambda})+\bar{\lambda}-\frac{1}{\sqrt{p}} \rho\right\|^{2}} \mathbf{z}^{w(\alpha+\rho+\widehat{\lambda})-\rho}}{\Delta(z)} \\
& =\frac{1}{\eta(\tau)^{\operatorname{rank}(Q)}} \sum_{w \in W} \sum_{\alpha \in Q \cap P^{+}} \sum_{w^{\prime} \in W}(-1)^{\ell(w)} \frac{q^{\frac{1}{2}\left\|\sqrt{p} w^{\prime}(\alpha+\rho+\widehat{\lambda})+\bar{\lambda}-\frac{1}{\sqrt{p}} \rho\right\|^{2}} \mathbf{z}^{w w^{\prime}(\alpha+\rho+\widehat{\lambda})-\rho}}{\Delta(z)} \\
& =\frac{1}{\eta(\tau)^{\operatorname{rank}(Q)}} \sum_{\alpha \in Q \cap P^{+}} \sum_{w^{\prime} \in W} \sum_{t \in W}(-1)^{\ell(t)+\ell\left(w^{\prime}\right)} \frac{q^{\frac{1}{2}\left\|\sqrt{p} w^{\prime}(\alpha+\rho+\widehat{\lambda})+\bar{\lambda}-\frac{1}{\sqrt{p}} \rho\right\|^{2}} \mathbf{z}^{t(\alpha+\rho+\widehat{\lambda})-\rho}}{\Delta(z)} \\
& =\frac{1}{\eta(\tau)^{\operatorname{rank}(Q)}} \sum_{\alpha \in Q \cap P^{+}} \sum_{w \in W}(-1)^{\ell(w)} q^{\frac{1}{2}\left\|\sqrt{p} w(\alpha+\rho+\widehat{\lambda})+\bar{\lambda}-\frac{1}{\sqrt{p}} \rho\right\|^{2}} \chi_{\widehat{\lambda}+\alpha}(z) \\
& =\sum_{\alpha \in Q \cap P^{+}} \chi_{\widehat{\lambda}+\alpha}(z)\left(\sum_{w \in W}(-1)^{\ell(w)} \frac{q^{\frac{1}{2}\left\|\sqrt{p} w(\alpha+\rho+\widehat{\lambda})+\bar{\lambda}-\frac{1}{\sqrt{p}} \rho\right\|^{2}}}{\eta(\tau)^{\operatorname{rank}(Q)}}\right) .
\end{aligned}
$$


The sum in the parenthesis is also the character of an affine $W$-algebra module [15, 18]. After we take the limit $z \rightarrow 1$, we get

$$
\operatorname{ch}\left[W(p, \lambda)_{Q}\right](\tau)=\sum_{\alpha \in Q \cap P^{+}} \operatorname{dim}(V(\widehat{\lambda}+\alpha))\left(\sum_{w \in W}(-1)^{\ell(w)} \frac{q^{\frac{1}{2}\left\|\sqrt{p} w(\alpha+\rho+\widehat{\lambda})+\bar{\lambda}-\frac{1}{\sqrt{p}} \rho\right\|^{2}}}{\eta(\tau)^{\operatorname{rank}(Q)}}\right),
$$

where $V(\gamma)$ denote the finite-dimensional $\mathfrak{g}$-module of highest weight $\gamma$. The previous formula is perhaps useful for representation theoretic consideration while equation (4.2) is more suitable for studying modular properties. If $\bar{\lambda}=0$, the sum over the Weyl group can be further simplified as

$$
\operatorname{ch}\left[W(p, \lambda)_{Q}\right](\tau)=\frac{1}{\eta(\tau)^{\operatorname{rank}(Q)}} \sum_{\alpha \in Q \cap P^{+}} \operatorname{dim}(V(\widehat{\lambda}+\alpha)) q^{\frac{1}{2 p}\|p(\alpha+\rho+\widehat{\lambda})-\rho\|^{2}} \prod_{\gamma \in \Delta_{+}}\left(1-q^{(\gamma, \alpha+\rho+\widehat{\lambda})}\right),
$$

after an application of the Weyl denominator formula.

Remark 3. Observe that, by Weyl's dimension formula, $\operatorname{dim} V(\gamma)$ depends polynomially on the labels $m_{j}$ of the highest weight $\gamma=m_{1} \omega_{1}+\cdots+m_{n} \omega_{n}$. The degree of this polynomial is $\left|\Delta_{+}\right|$.

Next, we are interested in modular transformation properties of $W(p)_{Q}$-characters. Let us first consider the $A_{1}$ case. Here $Q=\mathbb{Z} \alpha$ and $P=\mathbb{Z} \omega$. Two representatives of $Q^{0} / Q$ are 0 and $\omega$. Then $\widehat{\lambda}$ is either 0 or $\omega$, and the corresponding $2 p$ representatives of $L^{0} / L$ are given by

$$
\lambda=\frac{j}{\sqrt{p}} \omega
$$

where $j=-(p-1), \ldots, 0$, and $j=1, \ldots, p$, respectively. Clearly, $P^{+} \cap Q=\mathbb{N} \alpha=2 \mathbb{N} \omega$ and $\operatorname{dim}(V(2 n \omega))=2 n+1, \operatorname{dim}(V((2 n-1) \omega))=2 n, \rho=\omega$, and $W=\{ \pm 1\}$. There are two series of characters: For $-(p-1) \leq j \leq 0(\widehat{\lambda}=0)$, we have

$$
\operatorname{ch}\left[W\left(p, \frac{j \omega}{\sqrt{p}}\right)_{A_{1}}\right](\tau)=\frac{1}{\eta(\tau)} \sum_{n=0}^{\infty}(2 n+1)\left(q^{p\left(n+\frac{p-j-1}{2 p}\right)^{2}}-q^{p\left(n+\frac{p+j+1}{2 p}\right)^{2}}\right),
$$

while for $1 \leq j \leq p(\widehat{\lambda}=\omega)$, we have

$$
\operatorname{ch}\left[W\left(p, \frac{j \omega}{\sqrt{p}}\right)_{A_{1}}\right](\tau)=\frac{1}{\eta(\tau)} \sum_{n=0}^{\infty} 2 n\left(q^{p\left(n+\frac{2 p-j-1}{2 p}\right)^{2}}-q^{p\left(n+\frac{2 p+j+1}{2 p}\right)^{2}}\right) .
$$

These formulas are well known character formulas for the triplet vertex algebra modules [19] (see also [2] and references therein).

It is not hard to see that both families of characters can be written as linear combinations of $\frac{\theta_{p, j}(\tau)}{\eta(\tau)}$ and $\frac{\theta_{p, j}^{\prime}(\tau)}{\eta(\tau)}$, where

$$
\theta_{p, j}(\tau):=\sum_{n \in \mathbb{Z}} q^{\frac{1}{4 p}(2 p n+j)^{2}}
$$

are Jacobi theta functions and $\theta_{p, j}^{\prime}(\tau):=\sum_{n \in \mathbb{Z}}(2 p n+j) q^{(2 p n+j)^{2} /(4 p)}$ denote their Jacobi derivatives; see $[2,19]$ for more details. Consequently, the modular closure of the characters given in (4.4) and (4.5) is finite-dimensional with a basis given by

$$
\frac{\theta_{p, \ell}(\tau)}{\eta(\tau)}, 0 \leq \ell \leq p, \frac{\theta_{p, j}^{\prime}(\tau)}{\eta(\tau)}, 1 \leq j \leq p-1, \tau \frac{\theta_{p, k}^{\prime}(\tau)}{\eta(\tau)}, 1 \leq k \leq p .
$$

Although we do not have a similar explicit description of the modular closure of $W(p)_{Q}$-characters, we are able to prove a modularity result for all root lattices $Q$, except possibly $E_{8}$. For this purpose, we use results from [33]. Let $A \in \operatorname{Pos}(n ; \mathbb{Z})$ be even and $P \in \mathbb{C}\left[v_{1}, \ldots, v_{n}\right]$ be a harmonic polynomial 
with respect to $A^{-1}$ (as defined in Appendix A, Definition 1) of homogenous degree $d$. We define a theta-like function associated to $A, P$, and $\lambda \in A^{-1} \mathbb{Z}^{n}$ by

$$
\theta_{A, P, \lambda}(\tau):=\sum_{r \in \lambda+\mathbb{Z}^{n}} P(r) q^{\frac{1}{2} r^{\top} A r} .
$$

Then the transformations from [33, Section 2] can be summarized as

$$
\begin{aligned}
& \theta_{A, P, \lambda}(\tau+1)=e^{\pi i\left(\lambda^{\top} A \lambda\right)} \theta_{A, P, \lambda}(\tau), \\
& \theta_{A, P, \lambda}\left(-\frac{1}{\tau}\right)=\frac{(-i)^{d}(-i \tau)^{\frac{n}{2}+d}}{\sqrt{\operatorname{det}(A)}} \sum_{\mu \in A^{-1} \mathbb{Z}^{n} / \mathbb{Z}^{n}} e^{2 \pi i\left(\lambda^{\top} A \mu\right)} \theta_{A, P, \mu}(\tau) .
\end{aligned}
$$

Theorem 4.1. Let $Q$ be an $A D E$ type root lattice, except possibly $E_{8}$ (in which case we only have numerical evidence). Then each character $\operatorname{ch}\left[W(p, \lambda)_{Q}\right](\tau)$ is a sum of modular forms of non-negative integral weight. Moreover, the modular closure of $\operatorname{ch}\left[W(p, \lambda)_{Q}\right](\tau), \lambda \in L^{0} / L$, is finite-dimensional.

Proof: We first note that $\theta_{A, P, \mu}(\tau)$ transforms the same way as $\theta_{A, 1, \mu}(\tau)$, except for the additional multiplicative factor $(-i \tau)^{d}$ appearing in (4.7). But $\theta_{A, 1, \mu}(\tau)$ is an ordinary theta function which is known to be modular (of a certain level and multiplier), and therefore $\theta_{A, P, \mu}(\tau)$ is also modular.

It suffices to show that each irreducible character can be expressed as a linear combination of $\frac{\theta_{A, P, \mu}(\tau)}{\eta(\tau)^{n}}$, where the matrix $A$ is fixed and where $P$ belongs to a finite set of homogeneous harmonic polynomials. Indeed, provided that this holds, by using relations (4.6) and (4.7) we easily infer that the closure of the given set of characters is contained inside the $\mathrm{SL}_{2}(\mathbb{Z})$-invariant space spanned by $\tau^{j} \frac{\theta_{A, P, \mu}(\tau)}{\eta(\tau)^{n}}$, where $0 \leq j \leq \operatorname{deg}(P), \mu \in A^{-1} \mathbb{Z}^{n} / \mathbb{Z}^{n}$, and $P$ vary through the same finite set. This vector space is clearly finite-dimensional. Now we analyze $\operatorname{ch}\left[W(p, \lambda)_{Q}\right](\tau)$. By Lemma 6.1, combined with (4.2), we see that with $A=p B \in \operatorname{Pos}(n ; \mathbb{Z})$, where $B$ is the Gram matrix of $Q$, the expression $\eta(\tau)^{n} \operatorname{ch}\left[W(p, \lambda)_{Q}\right](\tau)$ can be written as a linear combination of $\theta_{A, P, \mu}$, $\mu \in A^{-1} \mathbb{Z}^{n} / \mathbb{Z}^{n}$, and where $P$ are certain polynomials. Next we show that all $P$ that appears in these linear combinations are harmonic with respect to $A^{-1}$. For this, it is sufficient to prove that $\prod_{\alpha \in \Delta_{+}}\left\langle x_{1} \alpha_{1}+\cdots+x_{n} \alpha_{n}+\widetilde{\mu}, \alpha\right\rangle \in \mathbb{C}\left[x_{1}, \ldots, x_{n}\right]$ is harmonic with respect to $A^{-1}$ for any "shift" $\tilde{\mu}$. This is immediate from Lemma A.3, Theorem A.4, Theorem A.6 and Remark 10 (thus we have to exclude $E_{8}$ ). It remains to show that $\theta_{A, P, \mu}$, where $P$ is harmonic with respect to $A^{-1}$, can be written as a linear combination of $\theta_{A, P, \mu}$, where $P$ is homogeneous. Clearly, every harmonic polynomial $P$ can be written as a sum of homogeneous harmonic polynomials and this proves the previous claim and the theorem.

For $Q=A_{1}$, it was shown in [3] that the relation $\left(L(0)-L_{s s}(0)\right)^{2}=0$ holds on any logarithmic $W(p)_{A_{1}}$-module, where $L_{s s}(0)$ denotes the semisimple part of the Virasoro operator $L(0)$. Existence of a non-trivial $L(0)$ Jordan block is closely tied to the fact that $\theta_{p, j}^{\prime}(\tau) / \eta(\tau)$, modular form of weight one, appears in the modular closure of irreducible characters. Observe that the proof of Theorem 4.1 implies that all irreducible characters contain $\theta_{A, P, \lambda}(\tau) / \eta(\tau)^{n}$ where $\operatorname{deg}(P):=\left|\Delta_{+}\right|$, where $\Delta_{+}$ is the set of positive roots (cf. Remark 3). Based on this observation we make the following

Conjecture 2. We have $\left(L(0)-L_{s s}(0)\right)^{\left|\Delta_{+}\right|+1}=0$ on any logarithmic $W(p)_{Q^{-} \text {module. In other }}$ words, $L(0)$ admits Jordan blocks of size at most $\left|\Delta_{+}\right|+1$.

4.3. Example: modular transformations for the character of $W(p)_{A_{2}}$. Here we examine the $Q=A_{2}$ case, that is $\mathfrak{g}=s \ell(3)$. Let $\left\{\alpha_{1}, \alpha_{2}\right\}$ be simple roots of $A_{2}$. The entries of the Cartan matrix are given by: $\left(\alpha_{1}, \alpha_{1}\right)=\left(\alpha_{2}, \alpha_{2}\right)=2$ and $\left(\alpha_{1}, \alpha_{2}\right)=\left(\alpha_{2}, \alpha_{1}\right)=-1$. For simplicity, we only 
consider the case $\lambda=\widehat{\lambda}=0$. We have

$$
\operatorname{dim}\left(V\left(m_{1} \omega_{1}+m_{2} \omega_{2}\right)\right)=\frac{\left(m_{1}+1\right)\left(m_{2}+1\right)\left(m_{1}+m_{2}+2\right)}{2}
$$

and by the Weyl character formula

$$
\operatorname{dim}\left(V\left(m_{1} \omega_{1}+m_{2} \omega_{2}\right)_{0}\right)=\min \left\{m_{1}, m_{2}\right\}+1 .
$$

For $\alpha=m_{1} \alpha_{1}+m_{2} \alpha_{2}$, with $m_{1}, m_{2} \in \mathbb{Z}$, we have

$$
\lim _{z \rightarrow 1}\left(\sum_{w \in S_{3}}(-1)^{\ell(w)} \frac{\mathbf{z}^{w(\alpha+\rho)-\rho}}{\Delta(z)}\right)=\frac{\left(2 m_{1}-m_{2}+1\right)\left(2 m_{2}-m_{1}+1\right)\left(m_{1}+m_{2}+2\right)}{2} .
$$

We now present two formulas for $\operatorname{ch}\left[W(p)_{A_{2}}\right](\tau)$. Formula (4.2), after $\mathbf{z} \rightarrow 1$, yields, after shifting $m_{j} \mapsto m_{j}-1$

$\operatorname{ch}\left[W(p)_{A_{2}}\right](\tau)=\sum_{\left(m_{1}, m_{2}\right) \in \mathbb{Z}^{2}}\left(2 m_{1}-m_{2}\right)\left(2 m_{2}-m_{1}\right)\left(m_{1}+m_{2}\right) \frac{q^{p\left(\left(m_{1}-\frac{1}{p}\right)^{2}+\left(m_{2}-\frac{1}{p}\right)^{2}-\left(m_{1}-\frac{1}{p}\right)\left(m_{2}-\frac{1}{p}\right)\right)}}{2 \eta(\tau)^{2}}$.

Similarly, relation (4.3), easily gives another expression for $\operatorname{ch}\left[W(p)_{A_{2}}\right]$ :

$$
\begin{gathered}
\sum_{\substack{m_{1}, m_{2}=0 \\
m_{1} \equiv m_{2}(\bmod 3)}}^{\infty} m_{1} m_{2}\left(m_{1}+m_{2}\right) \frac{q^{t_{p}\left(m_{1}-\frac{1}{p}, m_{2}-\frac{1}{p}\right)}\left(1-q^{m_{1}}\right)\left(1-q^{m_{2}}\right)\left(1-q^{m_{1}+m_{2}}\right)}{2 \eta(\tau)^{2}}, \text { where } \\
t_{p}\left(m_{1}, m_{2}\right):=\frac{p}{3}\left(m_{1}^{2}+m_{2}^{2}+m_{1} m_{2}\right) .
\end{gathered}
$$

Because we are interested modular properties of $\operatorname{ch}\left[W(p)_{A_{2}}\right](\tau)$ we shall focus on the first formula. If we let

$$
P\left(m_{1}, m_{2}\right):=\left(2 m_{1}-m_{2}\right)\left(2 m_{2}-m_{1}\right)\left(m_{1}+m_{2}\right)
$$

then

$$
B_{p}:=p\left(\begin{array}{cc}
2 & -1 \\
-1 & 2
\end{array}\right), \quad \quad P_{p}\left(m_{1}, m_{2}\right):=P\left(m_{1}+\frac{1}{p}, m_{2}+\frac{1}{p}\right), \quad \lambda_{p}:=-\frac{1}{p}(1,1)^{\top} .
$$

Then we have

$$
\begin{aligned}
& 2 \eta(\tau)^{2} \sum_{\left(m_{1}, m_{2}\right) \in \mathbb{Z}^{2}}\left(2 m_{1}-m_{2}\right)\left(2 m_{2}-m_{1}\right)\left(m_{1}+m_{2}\right) q^{p\left(\left(m_{1}-\frac{1}{p}\right)^{2}+\left(m_{2}-\frac{1}{p}\right)^{2}-\left(m_{1}-\frac{1}{p}\right)\left(m_{2}-\frac{1}{p}\right)\right)} \\
& =2 \eta(\tau)^{2} \sum_{\left(n_{1}, n_{2}\right) \in\left(-\frac{1}{p},-\frac{1}{p}\right)+\mathbb{Z}^{2}}\left(2 n_{1}-n_{2}+\frac{1}{p}\right)\left(2 n_{2}-n_{1}+\frac{1}{p}\right)\left(n_{1}+n_{2}+\frac{2}{p}\right) q^{p\left(n_{1}^{2}+n_{2}^{2}-n_{1} n_{2}\right)} \\
& =2 \eta(\tau)\left(\sum_{n=\left(n_{1}, n_{2}\right) \in\left(-\frac{1}{p},-\frac{1}{p}\right)+\mathbb{Z}^{2}}\left(2 n_{1}-n_{2}\right)\left(2 n_{2}-n_{1}\right)\left(n_{1}+n_{2}\right) q^{\frac{1}{2} n^{T} B_{p} n}+\frac{2}{p^{3}} \sum_{n \in\left(-\frac{1}{p},-\frac{1}{p}\right)+\mathbb{Z}^{2}} q^{\frac{1}{2} n^{T} B_{p} n}\right. \\
& \left.-\frac{3}{p} \sum_{n \in\left(-\frac{1}{p},-\frac{1}{p}\right)+\mathbb{Z}^{2}}\left(n_{1}^{2}-4 n_{1} n_{2}+n_{2}^{2}\right) q^{\frac{1}{2} n^{T} B_{p} n}+\frac{3}{p^{2}} \sum_{n \in\left(-\frac{1}{p},-\frac{1}{p}\right)+\mathbb{Z}^{2}}\left(n_{1}+n_{2}\right) q^{\frac{1}{2} n^{T} B_{p} n}\right) .
\end{aligned}
$$


Now each polynomial above is homogeneous and harmonic with respect to $B_{p}^{-1}$ (use Example 3 and Remark 8) so our character is a linear combination of $\theta_{P, B_{p}, \lambda}$ and thus formula (4.7) can be applied.

Remark 4. Alternatively, one can use (3) to obtain modular transformation formulas of $W(p)_{A_{2}{ }^{-}}$ characters.

\section{Characters of $W^{0}(p)_{Q}$-Modules}

5.1. Characters as constant terms. Next we discuss irreducible $W^{0}(p)_{Q}$-modules. In parallel to $[10,11]$, we are primarily interested in atypical irreducibles. Typical irreducibles are isomorphic to Fock spaces so their structure and character formulas are well-known (see Section 8). Because the vertex operator algebra $W^{0}(p)_{Q}$ is a subalgebra of the Fock space, it automatically admits infinitely many irreducible modules. In particular, infinitely many modules are obtained by restriction from $W(p)_{Q}$-modules - those are realized inside the lattice vertex algebra module. This way we obtain $W^{0}(p)_{Q^{-}}$-modules parametrized by $\beta \in L^{0}$ which we denote by $W^{0}(p, \beta)_{Q}$. Again, motivated by the singlet case, we assume that all such $W^{0}(p)_{Q}$-modules are irreducible (i.e., we conjecture that $W(p, \lambda)_{Q}$ is completely reducible as $W^{0}(p)_{Q}$-module) and that all atypical modules arise this way (cf. Remark 1). With these assumptions, we can easily compute characters of all atypical by using the full character $\operatorname{ch}\left[W(p, \lambda)_{Q}\right](\tau, z)$ as follows. Let $\beta \in L^{0}$ and $\beta \mapsto \lambda$ under the natural map $L^{0} \rightarrow L^{0} / L$, where $\lambda$ is one of the representatives fixed earlier. Observe that $\mathbf{z}$-powers in $\operatorname{ch}\left[W(p, \lambda)_{Q}\right](\tau, z)$ are contained in the set $\widehat{\lambda}+Q \subset P$. Moreover, because $\beta=\lambda+\sqrt{p} \gamma$, where $\gamma \in Q$, we extract the coefficient of $\mathbf{z}^{\beta^{\prime}}, \beta^{\prime}=\widehat{\lambda}+\gamma$, expressed as a constant term:

$$
\operatorname{ch}\left[W^{0}(p, \beta)_{Q}\right](\tau)=\mathrm{CT}_{\mathbf{z}}\left\{\mathbf{z}^{-\beta^{\prime}} \operatorname{ch}\left[W(p, \lambda)_{Q}\right](\tau, z)\right\} .
$$

The most prominent case here is $\beta=0$, where $\lambda=\widehat{\lambda}=0$, which gives the character of the vertex algebra $W^{0}(p)_{Q}$ (also discussed in the introduction)

$\operatorname{ch}\left[W^{0}(p)_{Q}\right](\tau)=\mathrm{CT}_{\mathbf{z}}\left\{\operatorname{ch}\left[W(p)_{Q}\right](\tau, z)\right\}=\sum_{\alpha \in Q \cap P^{+}} \operatorname{dim}\left(V(\alpha)_{0}\right)\left(\sum_{w \in W}(-1)^{\ell(w)} \frac{q^{\frac{1}{2}\left\|\sqrt{p} w(\alpha+\rho)-\frac{1}{\sqrt{p}} \rho\right\|^{2}}}{\eta(\tau)^{\operatorname{rank}(Q)}}\right)$,

where $V(\alpha)_{0}$ denotes the zero weight subspace of $V(\alpha)$. Unfortunately, not much is known about the dimension of $V(\alpha)_{0}$ for general $\mathfrak{g}$ and $\alpha$ (let alone any explicit formulas!).

Example 2. $\left(Q=A_{1}\right)$. We illustrate how this setup recovers characters studied in our earlier work [10]. Following the previous notation adopted for $A_{1}$, for $-(p-1) \leq j \leq 0, \widehat{\lambda}=0, k \in \mathbb{Z}$, we get

$$
\operatorname{ch}\left[W^{0}\left(p, \frac{j \omega}{\sqrt{p}}+k \sqrt{p} \alpha\right)_{A_{1}}\right](\tau)=\frac{1}{\eta(\tau)} \sum_{n \geq k}^{\infty}\left(q^{p\left(n+\frac{p-j-1}{2 p}\right)^{2}}-q^{p\left(n+\frac{p+j+1}{2 p}\right)^{2}}\right),
$$

while for $1 \leq j \leq p, \widehat{\lambda}=\omega, k \in \mathbb{Z}$, we obtain

$$
\operatorname{ch}\left[W^{0}\left(p, \frac{j \omega}{\sqrt{p}}+k \sqrt{p} \alpha\right)_{A_{1}}\right](\tau)=\frac{1}{\eta(\tau)} \sum_{n \geq k}^{\infty}\left(q^{p\left(n+\frac{2 p-j-1}{2 p}\right)^{2}}-q^{p\left(n+\frac{2 p+j+1}{2 p}\right)^{2}}\right) .
$$

In particular, we have

$$
\operatorname{ch}\left[W^{0}(p)_{A_{1}}\right](\tau)=\frac{1}{\eta(\tau)} \sum_{n=0}^{\infty}\left(q^{p\left(n+\frac{p-1}{2 p}\right)^{2}}-q^{p\left(n+\frac{p+1}{2 p}\right)^{2}}\right) .
$$


Remark 5. Observe that the numerator of $\operatorname{ch}\left[W^{0}(p)_{A_{1}}\right](\tau)$, studied extensively in [10, 11], is closely related to Roger's false theta function. Therefore it seems interesting to consider

$$
F_{Q, p}(\tau):=\eta(\tau)^{\operatorname{rank}(Q)} \operatorname{ch}\left[W^{0}(p)_{Q}\right](\tau) .
$$

From the Lie theoretic point of view, this can be regarded as a "higher rank" generalization of the classical false theta function. It would be especially interesting to understand these objects from number theoretic perspective.

5.2. Explicit example: the character of $W^{0}(p)_{A_{2}}$. Now we consider the character of $W^{0}(p)_{A_{2}}$. Observe from the definition

$$
\begin{aligned}
& \eta(\tau)^{2} \operatorname{ch}\left[W(p)_{A_{2}}\right](\tau, z)=\sum_{m, n \in \mathbb{Z}} \frac{q^{p(m+1)^{2}+p(n+1)^{2}-p(m+1)(n+1)-m-n-2+\frac{1}{p}}}{\left(1-z_{1}^{-1}\right)\left(1-z_{2}^{-1}\right)\left(1-z_{1}^{-1} z_{2}^{-1}\right)} \\
& \quad \times\left(z_{1}^{m} z_{2}^{n}-z_{1}^{-m+n-1} z_{2}^{n}-z_{1}^{m} z_{2}^{-n+m-1}+z_{1}^{-n-2} z_{2}^{-n+m-1}+z_{1}^{-m+n-1} z_{2}^{-m-2}-z_{1}^{-n-2} z_{2}^{-m-2}\right) \\
& \quad=\sum_{m, n \in \mathbb{Z}} \frac{q^{p\left(\left(m-\frac{1}{p}\right)^{2}+\left(n-\frac{1}{p}\right)^{2}-\left(m-\frac{1}{p}\right)\left(n-\frac{1}{p}\right)\right)}}{\left(1-z_{1}^{-1}\right)\left(1-z_{2}^{-1}\right)\left(1-z_{1}^{-1} z_{2}^{-1}\right)} \\
& \times\left(z_{1}^{m-1} z_{2}^{n-1}-z_{1}^{-m+n-1} z_{2}^{n-1}-z_{1}^{m-1} z_{2}^{-n+m-1}+z_{1}^{-n-1} z_{2}^{-n+m-1}+z_{1}^{-m+n-1} z_{2}^{-m-1}-z_{1}^{-n-1} z_{2}^{-m-1}\right) .
\end{aligned}
$$

To obtain a formula for $\operatorname{ch}\left[W^{0}(p)_{A_{2}}\right]$, we have to extract the constant term. After a straightforward computation, we get

Proposition 5.1. We have

$$
\operatorname{ch}\left[W^{0}(p)_{A_{2}}\right](\tau)=\frac{1}{\eta(\tau)^{2}} \sum_{\substack{m_{1}, m_{2}=1 \\ m_{1} \equiv m_{2}(\bmod 3)}}^{\infty} \min \left(m_{1}, m_{2}\right) q^{t_{p}\left(m_{1}-\frac{1}{p}, m_{2}-\frac{1}{p}\right)}\left(1-q^{m_{1}}\right)\left(1-q^{m_{2}}\right)\left(1-q^{m_{1}+m_{2}}\right),
$$

where $t_{p}\left(m_{1}, m_{2}\right)$ is defined in (4.8).

\section{Asymptotic EXPANSiOnS}

In this part, we derive asymptotic expansions of characters $\operatorname{ch}\left[W^{0}(p, \beta)\right](\tau)$.

Lemma 6.1. Let $\lambda \in P^{+}$. Then

$$
\lim _{z \rightarrow 1}\left(\sum_{w \in W}(-1)^{\ell(w)} \frac{\mathbf{z}^{w\left(\frac{\lambda+\rho}{p}\right)-\rho}}{\Delta(z)}\right)=\frac{1}{p^{\left|\Delta_{+}\right|}} \prod_{\alpha \in \Delta^{+}} \frac{\langle\lambda+\rho, \alpha\rangle}{\langle\rho, \alpha\rangle}=\frac{1}{p^{\left|\Delta_{+}\right|}} \operatorname{dim}(V(\lambda)) .
$$

Proof: Since the proof is analogous to the proof of Weyl's dimension formula, we omit it here.

Theorem 6.2. Let $\beta \in L^{0}$, whose coset representative in $L^{0} / L$ is $\lambda$. Then, as $t \rightarrow 0^{+}$, we have

$$
\eta(i t)^{\operatorname{dim}(Q)} \operatorname{ch}\left[W^{0}(p, \beta)_{Q}\right](i t) \sim \frac{\operatorname{dim}(V(-\sqrt{p} \bar{\lambda}))}{p^{\left|\Delta_{+}\right|}}+O(\sqrt{t}),
$$

where $\bar{\lambda}=\sum_{j=1}^{n}\left(1-s_{j}\right) \frac{\omega_{j}}{\sqrt{p}}$ as in Section 4.2. In particular,

$$
\begin{aligned}
& \eta(i t)^{\operatorname{rank}(Q)} \operatorname{ch}\left[W^{0}(p)_{Q}\right](i t) \sim \frac{1}{p^{\left|\Delta_{+}\right|}}+O(\sqrt{t}), \text { where } \\
&\left|\Delta_{+}\right|:=\left\{\begin{array}{cl}
\frac{n(n+1)}{2} & \text { if } Q=A_{n}, \\
n(n-1) & \text { if } Q=D_{n}, \\
36 & \text { if } Q=E_{6}, \\
63 & \text { if } Q=E_{7}, \\
120 & \text { if } Q=E_{8} .
\end{array}\right.
\end{aligned}
$$


Proof: First we choose $\lambda$ to be a representative in $L^{0} / L$, as fixed above. Next we rewrite

$$
\begin{aligned}
& \eta(\tau)^{\operatorname{dim}(Q)} \operatorname{ch}\left[W(p, \lambda)_{Q}\right](\tau, z)=\sum_{\beta \in Q} \sum_{w \in W}(-1)^{\ell(w)} q^{\frac{1}{2}\left\|\sqrt{p} \beta+w\left(\lambda+\alpha_{0} \rho\right)\right\|^{2}} z^{\beta+w(\widehat{\lambda}+\rho)-\rho} \\
& =\sum_{\beta \in Q} \sum_{w \in W}(-1)^{\ell(w)} q^{\frac{p}{2}\|\beta\|^{2}+\frac{1}{2}\left\|\lambda+\rho \alpha_{0}\right\|^{2}+\sqrt{p}\left(\beta, \omega\left(\lambda+\rho \alpha_{0}\right)\right)} z^{\beta+w(\widehat{\lambda}+\rho)-\rho} \\
& =\sum_{w \in W}(-1)^{\ell(w)} q^{\frac{1}{2}\left\|\lambda+\rho \alpha_{0}\right\|^{2}} \sum_{\beta \in Q} e^{\pi i \tau p\|\beta\|^{2}+2 \pi i\left(\beta, \sqrt{p} \tau w\left(\lambda+\rho \alpha_{0}\right)+\mathbf{u}\right)} z^{w(\widehat{\lambda}+\rho)-\rho} \\
& =q^{\frac{1}{2}\left\|\lambda+\rho \alpha_{0}\right\|^{2}} \sum_{w \in W}(-1)^{\ell(w)} \Theta_{0, \sqrt{p} \tau w\left(\lambda+\rho \alpha_{0}\right)+\mathbf{u}}\left(p \tau ; A_{Q}\right) z^{w(\widehat{\lambda}+\rho)-\rho} \\
& =q^{\frac{1}{2}\left\|\lambda+\rho \alpha_{0}\right\|^{2}} \sum_{w \in W}(-1)^{\ell(w)} \frac{1}{\sqrt{\operatorname{det}\left(A_{Q}\right)}(-p i \tau)^{\frac{n}{2}}} \Theta_{-\sqrt{p} \tau w\left(\lambda+\rho \alpha_{0}\right)-\mathbf{u}, 0}\left(-\frac{1}{p \tau} ; A_{Q^{0}}\right) z^{w(\widehat{\lambda}+\rho)-\rho},
\end{aligned}
$$

where $\mathbf{u}=\left(u_{1}, \ldots, u_{n}\right), \alpha_{0}=\sqrt{p}-\frac{1}{\sqrt{p}}$, and $A_{Q}$ denotes the determinant of the Gram matrix of $Q$. In the last formula we have to exercise caution. As all vectors involved are in $\mathbb{C}^{n}$, the vector $w\left(\lambda+\rho \alpha_{0}\right)$ has to be written appropriately depending on whether the summation is over $Q$ or $Q^{0}$. For this purpose we use $\|\cdot\|_{0}$ if the summation is over $Q^{0}$. Recall (5.1), where we define $\operatorname{ch}\left[W^{0}(p, \lambda)_{Q}\right](\tau)$ as the constant term of the full characters $\operatorname{ch}\left[W(p, \lambda)_{Q}\right](\tau, z) z^{-\lambda}$. This expression involves integral powers of $z$, so we can write

$$
\eta(\tau)^{\operatorname{dim}(Q)} \operatorname{ch}\left[W^{0}(p, \lambda)_{Q}\right](\tau)=\eta(\tau)^{\operatorname{dim}(Q)} \int_{\left[-\frac{1}{2}, \frac{1}{2}\right]^{n}} \operatorname{ch}\left[W(p, \lambda)_{Q}\right](\tau, z) z^{-\lambda} \mathbf{d u}
$$

In each sum $\Theta_{-\sqrt{p} \tau w\left(\lambda+\rho \alpha_{0}\right)-\mathbf{u}, 0}\left(-\frac{1}{p \tau} ; Q^{0}\right)$, the zeroth term dominates so we have

$$
\begin{aligned}
q^{-\frac{1}{2}\left\|\lambda+\rho \alpha_{0}\right\|^{2}} & \eta(\tau)^{\operatorname{dim}(Q)} \operatorname{ch}\left[W^{0}(p, \lambda)_{Q}\right](\tau) \\
& \sim \sum_{w \in W} e^{-\pi i \tau\left\|\lambda+\rho \alpha_{0}\right\|_{0}^{2}} \int_{\left[-\frac{1}{2}, \frac{1}{2}\right]^{n}} e^{-\frac{\pi i}{p \tau}\left\|\sqrt{p} \tau w\left(\lambda+\rho \alpha_{0}\right)+\mathbf{u}\right\|_{0}^{2}} \frac{z^{w(\widehat{\lambda}+\rho)-\rho-\lambda}}{\Delta(z)} d \mathbf{u} \\
& =\sum_{w \in W} e^{-\pi i \tau\left\|\lambda+\rho \alpha_{0}\right\|_{0}^{2}} \int_{\left[-\frac{1}{2}, \frac{1}{2}\right]^{n}} e^{-\frac{\pi i}{p \tau}\left(2 \sqrt{p} \tau(w(\lambda+Q \rho), \mathbf{u})+\|\mathbf{u}\|_{0}^{2}\right)} \frac{z^{w(\widehat{\lambda}+\rho)-\rho-\lambda}}{\Delta(z)} d \mathbf{u} .
\end{aligned}
$$

We next turn the integral in (6.2) into an integral over $\mathbb{R}^{n}$ and show that this only introduces an exponentially small error. More generally, we prove that for $\alpha_{j} \in \mathbb{R}(j \in \mathbb{N}, j<n)$ and $M \in \operatorname{Pos}(n ; \mathbb{R})$

$$
\int_{(1, \infty)^{j} \times\left[-\frac{1}{2}, \frac{1}{2}\right]^{n-j}} e^{2 \pi i \sum_{j=1}^{n} \alpha_{j} u_{j}-\frac{1}{\tau} M[\mathbf{u}]} \mathbf{d u}
$$

is exponentially small. For this, we use the well-known fact that there exist $\alpha, \beta \in \mathbb{R}^{+}$such that $\alpha I_{n}<M<\beta I_{n}$, where $A>B$ for $A, B \in \operatorname{Pos}(n ; \mathbb{R})$ means there $A-B \in \operatorname{Pos}(n ; \mathbb{R})$. Thus (6.3) may be bounded against

$$
\ll \int_{(1, \infty)^{j} \times\left[-\frac{1}{2}, \frac{1}{2}\right]^{n-j}} e^{-\frac{\alpha}{\operatorname{Im}(\tau)} \sum_{j=1}^{n} u_{j}^{2}} \mathbf{d u}
$$

The claim follows since for $a \in \mathbb{R}^{+}, \int_{\mathbb{R}}^{\infty} e^{-\frac{a}{\operatorname{Im}(\tau)} u^{2}} d u$ is exponentially small. 
Returning to (6.2), we can now write

$$
\begin{aligned}
& q^{-\frac{1}{2}\left\|\lambda+\rho \alpha_{0}\right\|^{2}} \eta(\tau)^{\operatorname{dim}(Q)} \operatorname{ch}\left[W^{0}(p, \lambda)_{Q}\right](\tau) \sim \int_{\mathbb{R}^{n}} e^{-\frac{2 \pi i}{\sqrt{p}}\left(w\left(\lambda+\alpha_{0} \rho\right), \mathbf{u}\right)-\frac{\pi i\|\mathbf{u}\|_{0}^{2}}{p \tau}} \frac{z^{w(\widehat{\lambda}+\rho)-\rho-\lambda}}{\Delta(z)} \mathbf{d u} \\
& =\int_{\mathbb{R}^{n}} z^{-\frac{\pi i\|\mathbf{u}\|_{0}^{2}}{p \tau}-\frac{w\left(\lambda+\alpha_{0} \rho\right)}{\sqrt{p}}+w(\widehat{\lambda}+\rho)-\rho-\lambda} \frac{1}{\Delta(z)} \mathbf{d u}=\int_{\mathbb{R}^{n}} z^{-\frac{\pi i\|\mathbf{u}\|_{0}^{2}}{p \tau}+w\left(-\frac{\bar{\lambda}}{\sqrt{p}}+\frac{\rho}{p}\right)-\rho-\lambda} \frac{1}{\Delta(z)} \mathbf{d u} .
\end{aligned}
$$

Substituting $\tau=i t$ and summing the above expression over the Weyl group $W$, we obtain

$$
F(i t):=\int_{\mathbb{R}^{n}} e^{-\frac{\pi\|\mathbf{u}\|_{0}^{2}}{p t}} \frac{\sum_{w \in W}(-1)^{\ell(w)} z^{w\left(-\frac{\bar{\lambda}}{\sqrt{p}}+\frac{\rho}{p}\right)}}{z^{\rho} \Delta(z)} \mathbf{d u} .
$$

Observe that (by Lemma 6.1), as $\mathbf{u} \rightarrow 0$,

$$
\frac{\sum_{w \in W}(-1)^{\ell(w)} z^{w\left(-\frac{\bar{\lambda}}{\sqrt{p}}+\frac{\rho}{p}\right)}}{z^{\rho} \Delta(z)} \rightarrow \frac{\operatorname{dim}(V(-\sqrt{p} \bar{\lambda}))}{p^{\left|\Delta_{+}\right|}} .
$$

Next, we consider the $u$-expansion

$$
\frac{\sum(-1)^{\ell(w)} z^{w\left(-\frac{\bar{\lambda}}{\sqrt{p}}+\frac{\rho}{p}\right)-\lambda}}{z^{\rho} \Delta(z)}=\sum_{\left(m_{1}, \ldots, m_{n}\right) \in \mathbb{N}^{n}} \alpha\left(m_{1}, \ldots, m_{n}\right) u_{1}^{m_{1}} \cdots u_{n}^{m_{1}},
$$

with $\alpha(0, \ldots, 0)=\frac{\operatorname{dim}(V(-\sqrt{p} \bar{\lambda}))}{p^{|\Delta+|}}$. Substituting $v_{j}=u_{j} \sqrt{\frac{2 \pi}{t p}}$ and using (6.4), we get

$$
\begin{aligned}
F(i t) \sim & \sum_{\left(m_{1}, \ldots, m_{n}\right) \in \mathbb{N}^{n}} \int_{\mathbb{R}^{n}} e^{-\frac{\|\mathbf{v}\|_{0}^{2}}{2}}\left(\sqrt{\frac{t p}{2 \pi}}\right)^{n+\sum_{k=1}^{n} m_{k}} \alpha\left(m_{1}, \ldots, m_{n}\right) v_{1}^{m_{1}} \cdots v_{n}^{m_{1}} \mathbf{d} \mathbf{v} \\
= & \frac{\operatorname{dim}(V(-\sqrt{p} \bar{\lambda}))}{p^{\left|\Delta_{+}\right|}} \sqrt{\frac{(2 \pi)^{n}}{\operatorname{det}\left(Q^{0}\right)}}\left(\sqrt{\frac{t p}{2 \pi}}\right)^{n} \\
& +\sum_{\left(m_{1}, \ldots, m_{n}\right) \neq 0} \int_{\mathbb{R}^{n}} e^{-\frac{\|\mathbf{v}\|_{0}^{2}}{2}}\left(\sqrt{\frac{t p}{2 \pi}}\right)^{n+\sum_{k=1}^{n} m_{k}} \alpha\left(m_{1}, \ldots, m_{n}\right) v_{1}^{m_{1}} \cdots v_{n}^{m_{1}} \mathbf{d v}
\end{aligned}
$$

where we used the generalized Gaussian integral formula

$$
\int_{\mathbb{R}^{n}} e^{-\frac{1}{2} \sum_{j=1}^{n} \sum_{k=1}^{n} C_{j, k} v_{j} v_{k}} d v_{1} \cdots d v_{n}=\sqrt{\frac{(2 \pi)^{n}}{\operatorname{det}(C)}},
$$

where $C$ is any symmetric positive definite matrix. Taking into account that the prefactor

$$
e^{-\pi t|| \lambda+\alpha_{0} \rho\left\|^{2}+\pi t|| \lambda+\alpha_{0} \rho\right\|_{0}^{2}}
$$

does not contribute to the leading asymptotics, along with the formula (6.1), we get

$$
\begin{aligned}
& \eta(i t)^{\operatorname{rank}(Q)} \operatorname{ch}\left[W^{0}(p, \lambda)_{Q}\right](i t) \\
& \sim \frac{\operatorname{dim}(V(-\sqrt{p} \bar{\lambda}))}{p^{\left|\Delta_{+}\right|}}\left(\sqrt{\frac{t p}{2 \pi}}\right)^{n} \sqrt{\frac{(2 \pi)^{n}}{\operatorname{det}\left(A_{Q^{0}}\right)}} \frac{1}{(\sqrt{t p})^{n} \sqrt{\operatorname{det}\left(A_{Q}\right)}}+O(\sqrt{t})=\frac{\operatorname{dim}(V(-\sqrt{p} \bar{\lambda}))}{p^{\left|\Delta_{+}\right|}}+O(\sqrt{t}),
\end{aligned}
$$

as desired. Observe that for general $\lambda \in L^{0}$, the leading asymptotic only depends on its coset representative, so the proof follows. 
Remark 6. In general, the coefficients $\alpha\left(m_{1}, \ldots, m_{n}\right)$ are difficult to compute explicitly for all $\lambda$. However for $\hat{\lambda}=0$ the numerator in the Weyl character formula admits a product form so it is possible to get a more explicit asymptotic expansion. We do this in the next section for $Q=A_{2}$.

\section{EXPLiCit EXAMPLE: THE FUlL ASYMPtotic EXPANSION of $\eta(\tau)^{2} \operatorname{ch}\left[W^{0}(p)_{A_{2}}\right](\tau)$}

Observe that our method in Section 6 can in theory be used to compute a full asymptotic expansion. In this section we prove Theorem 1.2 from the introduction.

Proof of Theorem 1.2: As in the general case we may write

$$
f(\tau)=\eta(\tau)^{2} \mathrm{CT}_{z} \operatorname{ch}\left[W^{0}(p)_{A_{2}}\right](\tau, z)=\eta(\tau)^{2} \int_{\left[-\frac{1}{2}, \frac{1}{2}\right]^{2}} \operatorname{ch}\left[W^{0}(p)_{A_{2}}\right](\tau, z) d u_{1} d u_{2}
$$

with $z_{j}:=e^{2 \pi i u_{j}}$. Define

$$
\mathcal{G}(u, v ; \tau):=\sum_{m, n \in \mathbb{Z}} q^{p\left(m^{2}+n^{2}-m n\right)-m-n} e^{2 \pi i(m u+n v)} .
$$

Then

$$
\begin{aligned}
f(\tau)= & \int_{\left[-\frac{1}{2}, \frac{1}{2}\right]^{2}}\left(\mathcal{G}\left(u_{1}, u_{2} ; \tau\right)-\mathcal{G}\left(-u_{1}, u_{1}+u_{2} ; \tau\right)-\mathcal{G}\left(u_{1}+u_{2},-u_{2} ; \tau\right)+\mathcal{G}\left(u_{2},-u_{1}-u_{2} ; \tau\right)\right. \\
& \left.+\mathcal{G}\left(-u_{1}-u_{2}, u_{1} ; \tau\right)-\mathcal{G}\left(-u_{2},-u_{1} ; \tau\right)\right) \frac{z_{1}^{-1} z_{2}^{-1} q^{\frac{1}{p}}}{\left(1-z_{1}^{-1}\right)\left(1-z_{2}^{-1}\right)\left(1-z_{1}^{-1} z_{2}^{-1}\right)} d u_{1} d u_{2} .
\end{aligned}
$$

An application of (3.1) yields

$$
\mathcal{G}(u, v ; \tau)=\Theta_{0,\left(\begin{array}{l}
u-\tau \\
v-\tau
\end{array}\right)}\left(2 p \tau ;\left(\begin{array}{cc}
1 & -\frac{1}{2} \\
-\frac{1}{2} & 1
\end{array}\right)\right)=\frac{1}{\sqrt{3} p(-i \tau)} \Theta_{\left(\begin{array}{c}
\tau-u \\
\tau-v
\end{array}\right), 0}\left(-\frac{1}{2 p \tau} ; \frac{4}{3}\left(\begin{array}{cc}
1 & \frac{1}{2} \\
\frac{1}{2} & 1
\end{array}\right)\right) .
$$

Now

$$
\Theta_{\left(\begin{array}{c}
\tau-u \\
\tau-v
\end{array}\right), 0}\left(-\frac{1}{2 p \tau} ; \frac{4}{3}\left(\begin{array}{cc}
1 & \frac{1}{2} \\
\frac{1}{2} & 1
\end{array}\right)\right)=e^{-\frac{2 \pi i \tau}{p}} \sum_{n, m \in \mathbb{Z}} e^{-\frac{2 \pi i}{3 p \tau}\left((n-u)^{2}+(m-v)^{2}+(n-u)(m-v)\right)} e^{-\frac{2 \pi i}{p}((n-u)+m-v)} .
$$

We get exponentially small terms unless $n=u, m=v$. In particular in all cases that occur only the $n=m=0$ term contributes. Thus we may approximate the integrand in (7.1) by

$$
\begin{aligned}
& -\frac{z_{1} z_{2}}{\left(1-z_{1}\right)\left(1-z_{2}\right)\left(1-z_{1} z_{2}\right)} e^{-\frac{2 \pi i}{3 p \tau}\left(u_{1}^{2}+u_{2}^{2}+u_{1} u_{2}\right)} \\
& \times\left(e^{\frac{2 \pi i}{p}\left(u_{1}+u_{2}\right)}-e^{\frac{2 \pi i}{p} u_{2}}-e^{\frac{2 \pi i}{p} u_{1}}+e^{-\frac{2 \pi i}{p} u_{2}}+e^{-\frac{2 \pi i}{p} u_{1}}-e^{-\frac{2 \pi i}{p}\left(u_{1}+u_{2}\right)}\right) \frac{1}{\sqrt{3} p(-i \tau)} .
\end{aligned}
$$

Moreover, as before we introduce an exponentially small error if in (7.1) we integrate over $\mathbb{R}^{2}$. To compute the remaining integral, we set

$$
f(u, v, w):=-\frac{e^{\pi i(u+v+w)+\pi i \frac{(u+v+w)}{p}}\left(1-e^{-\frac{2 \pi i u}{p}}\right)\left(1-e^{-\frac{2 \pi i v}{p}}\right)\left(1-e^{-\frac{2 \pi i w}{p}}\right)}{\left(1-e^{2 \pi i u}\right)\left(1-e^{2 \pi i v}\right)\left(1-e^{2 \pi i w}\right)} .
$$

Note that $f$ has only removable singularities in all variables and we may hence write

$$
f(u, v, w)=: \sum_{n_{1}, n_{2}, n_{3} \geq 0} \alpha\left(n_{1}, n_{2}, n_{3}\right) u^{n_{1}} v^{n_{2}} w^{n_{3}} .
$$


Note that in particular $\alpha(0,0,0)=1 / p^{3}$. We then obtain, inserting (7.2) and (7.3) in (7.1),

$$
\begin{aligned}
f(i t) & \sim \frac{1}{\sqrt{3} p t} \int_{\mathbb{R}^{2}} f\left(u_{1}, u_{2}, u_{1}+u_{2}\right) e^{-\frac{2 \pi}{3 p t}\left(u_{1}^{2}+u_{2}^{2}+u_{1} u_{2}\right)} d u_{1} d u_{2} \\
& =\frac{1}{\sqrt{3} p t} \sum_{n_{1}, n_{2}, n_{3} \geq 0} \alpha\left(n_{1}, n_{2}, n_{3}\right) \int_{\mathbb{R}^{2}} u_{1}^{n_{1}} u_{2}^{n_{2}}\left(u_{1}+u_{2}\right)^{n_{3}} e^{-\frac{2 \pi}{3 p t}\left(u_{1}^{2}+u_{2}^{2}+u_{1} u_{2}\right)} d u_{1} d u_{2} \\
& =\frac{1}{\sqrt{3} p t} \sum_{n_{1}, n_{2}, n_{3} \geq 0} \alpha\left(n_{1}, n_{2}, n_{3}\right)\left(\sqrt{\frac{3 p t}{2 \pi}}\right)^{n_{1}+n_{2}+n_{3}+2} \int_{\mathbb{R}^{2}} e^{-\left(u_{1}^{2}+u_{2}^{2}+u_{1} u_{2}\right)} u_{1}^{n_{1}} u_{2}^{n_{2}}\left(u_{1}+u_{2}\right)^{n_{3}} d u_{1} d u_{2},
\end{aligned}
$$

where in the last equation we made the change of variables $u_{j} \mapsto u_{j} \sqrt{\frac{3 p t}{2 \pi}}$. To finish the proof we are left to compute $\alpha\left(n_{1}, n_{2}, n_{3}\right)$ and the remaining integral.

To determine $\alpha\left(n_{1}, n_{2}, n_{3}\right)$, recall that

$$
\frac{e^{\pi i u+\frac{\pi i u}{p}}\left(1-e^{-\frac{2 \pi i u}{p}}\right)}{1-e^{2 \pi i u}}=\sum_{n \geq 0}\left(B_{n}\left(\frac{1}{2}-\frac{1}{2 p}\right)-B_{n}\left(\frac{1}{2}+\frac{1}{2 p}\right)\right) \frac{(2 \pi i u)^{n-1}}{n !}
$$

with $B_{n}(x)$ the $n$-th Bernoulli polynomial. Since the left hand side is an even function, it follows that $n$ in the sum is odd. Moreover, noting that $B_{n}(1-x)=(-1)^{n} B_{n}(x)$, gives that (7.4) equals

$$
2 \sum_{n \geq 0} \frac{B_{2 n+1}\left(\frac{1}{2}-\frac{1}{2 p}\right)}{(2 n+1) !}(2 \pi i u)^{2 n}
$$

Thus $\alpha\left(n_{1}, n_{2}, n_{3}\right)=0$ unless $n_{1}, n_{2}$, and $n_{3}$ are even in which case we obtain

$$
\alpha\left(2 n_{1}, 2 n_{2}, 2 n_{3}\right)=-8 \frac{B_{2 n_{1}+1}\left(\frac{1}{2}-\frac{1}{2 p}\right)}{\left(2 n_{1}+1\right) !} \frac{B_{2 n_{2}+1}\left(\frac{1}{2}-\frac{1}{2 p}\right)}{\left(2 n_{2}+1\right) !} \frac{B_{2 n_{3}+1}\left(\frac{1}{2}-\frac{1}{2 p}\right)}{\left(2 n_{3}+1\right) !}(2 \pi i)^{2 n_{1}+2 n_{2}+2 n_{3}} .
$$

To finish the proof, define

$$
\beta\left(n_{1}, n_{2}, n_{3}\right):=\int_{\mathbb{R}^{2}} u_{1}^{2 n_{1}} u_{2}^{2 n_{2}}\left(u_{1}+u_{2}\right)^{2 n_{3}} e^{-\left(u_{1}^{2}+u_{2}^{2}+u_{1} u_{2}\right)} d u_{1} d u_{2} .
$$

We can evaluate (7.5) as follows: we set $u_{1}=t_{1}+\frac{t_{2}}{\sqrt{3}}, u_{2}=-t_{1}+\frac{t_{2}}{\sqrt{3}}$ and

$$
\begin{aligned}
& \beta\left(n_{1}, n_{2}, n_{3}\right)=\left(\frac{2}{\sqrt{3}}\right)^{2 n_{3}+1} \int_{\mathbb{R}^{2}}\left(t_{1}+\frac{t_{2}}{\sqrt{3}}\right)^{2 n_{1}}\left(t_{1}-\frac{t_{2}}{\sqrt{3}}\right)^{2 n_{2}} t_{2}^{2 n_{3}} e^{-t_{1}^{2}-t_{2}^{2}} d t_{1} d t_{2} \\
& =\frac{2^{2 n_{3}+1}}{3^{\frac{1}{2}+n_{1}+n_{2}+n_{3}}} \sum_{r=0}^{2 n_{1}} \sum_{j=0}^{2 n_{2}}\left(\begin{array}{c}
2 n_{1} \\
r
\end{array}\right)\left(\begin{array}{c}
2 n_{2} \\
j
\end{array}\right) 3^{r+j} \int_{\mathbb{R}^{2}} t_{1}^{r+j}(-1)^{j} t_{2}^{2 n_{1}+2 n_{2}+2 n_{3}-r-j} e^{-t_{1}^{2}-t_{2}^{2}} d t_{1} d t_{2} \\
& =\frac{2^{2 n_{3}+1}}{3^{\frac{1}{2}+n_{1}+n_{2}+n_{3}}} \sum_{k=0}^{n_{1}+n_{2}} \sum_{j=0}^{2 k}\left(\begin{array}{c}
2 n_{1} \\
2 k-j
\end{array}\right)\left(\begin{array}{c}
2 n_{2} \\
j
\end{array}\right)(-1)^{j} 3^{k} \int_{\mathbb{R}^{2}} t_{1}^{2 k} t_{2}^{2 n_{1}+2 n_{2}+2 n_{3}-2 k} e^{-t_{1}^{2}-t_{2}^{2}} d t_{1} d t_{2} \\
& =\frac{2^{2 n_{3}+1}}{3^{\frac{1}{2}+n_{1}+n_{2}+n_{3}}} \sum_{k=0}^{n_{1}+n_{2}} \sum_{j=0}^{2 k}\left(\begin{array}{c}
2 n_{1} \\
2 k-j
\end{array}\right)\left(\begin{array}{c}
2 n_{2} \\
j
\end{array}\right)(-1)^{j} 3^{k} \Gamma\left(k+\frac{1}{2}\right) \Gamma\left(n_{1}+n_{2}+n_{3}-k+\frac{1}{2}\right),
\end{aligned}
$$


where we used the substitution $r+j=2 k$ to eliminate summation indices for which the Gaussian integral is vanishing. This gives

$$
\begin{aligned}
f(i t) \sim-\frac{4}{\pi} \sum_{n_{1}, n_{2}, n_{3} \geq 0} & 2^{2 n_{3}+1}(-2 \pi p t)^{n_{1}+n_{2}+n_{3}} \frac{B_{2 n_{1}+1}\left(\frac{1}{2}-\frac{1}{2 p}\right)}{\left(2 n_{1}+1\right) !} \frac{B_{2 n_{2}+1}\left(\frac{1}{2}-\frac{1}{2 p}\right)}{\left(2 n_{2}+1\right) !} \frac{B_{2 n_{3}+1}\left(\frac{1}{2}-\frac{1}{2 p}\right)}{\left(2 n_{3}+1\right)} \\
& \times \sum_{k=0}^{n_{1}+n_{2}} \sum_{j=0}^{2 k}\left(\begin{array}{c}
2 n_{1} \\
2 k-j
\end{array}\right)\left(\begin{array}{c}
2 n_{2} \\
j
\end{array}\right)(-1)^{j} 3^{k} \Gamma\left(k+\frac{1}{2}\right) \Gamma\left(n_{1}+n_{2}+n_{3}-k+\frac{1}{2}\right) .
\end{aligned}
$$

The claim follows, setting

$$
\begin{aligned}
& \alpha(N):=-\frac{4}{\pi} \sum_{\substack{n_{1}, n_{2}, n_{3} \geq 0 \\
N=n_{1}+n_{2}+n_{3}}} 2^{2 n_{3}+1} \frac{B_{2 n_{1}+1}\left(\frac{1}{2}-\frac{1}{2 p}\right)}{\left(2 n_{1}+1\right) !} \frac{B_{2 n_{2}+1}\left(\frac{1}{2}-\frac{1}{2 p}\right)}{\left(2 n_{2}+1\right) !} \frac{B_{2 n_{3}+1}\left(\frac{1}{2}-\frac{1}{2 p}\right)}{\left(2 n_{3}+1\right) !} \\
& \times \sum_{k=0}^{n_{1}+n_{2}} \sum_{j=0}^{2 k}\left(\begin{array}{c}
2 n_{1} \\
2 k-j
\end{array}\right)\left(\begin{array}{c}
2 n_{2} \\
j
\end{array}\right)(-1)^{j} 3^{k} \Gamma\left(k+\frac{1}{2}\right) \Gamma\left(n_{1}+n_{2}+n_{3}-k+\frac{1}{2}\right) .
\end{aligned}
$$

Remark 7. Alternatively one could use a 2-dimensional version of the Euler-McLaurin summation formula as, for example, stated in [35]. However, this requires splitting the function of interest into many summands to which one may apply Euler-McLaurin which makes the calculation much more complicated than the use of Jacobi transformation properties.

\section{8. (Analytic) QuANTUm Dimensions of $W^{0}(p)_{Q^{-M O d u l e S}}$}

As we previously noted (cf. Remark 1), presently there is no complete classification of irreducible $W^{0}(p)_{Q}$-modules for $\operatorname{rank}(Q) \geq 2$. Nevertheless, there are strong indications that irreducible $W^{0}(p)_{Q}$-modules fall into two categories: typical and atypical.

(i) Typical modules are just rank $n$ Fock spaces $F_{\lambda}$, which are irreducible as $W^{0}(p)_{Q}$-modules. Typical characters are therefore given by

$$
\operatorname{ch}\left[F_{\lambda}\right](\tau)=\frac{q^{\frac{\left\|\lambda-\alpha_{0} \rho\right\|^{2}}{2}}}{\eta(\tau)^{n}},
$$

where $\lambda \notin L^{0}$ (see [12]).

(ii) Atypical modules, discussed above, form a discrete family parametrized by $\beta \in L^{0}$, and their characters are

$$
\operatorname{ch}\left[W^{0}(p, \beta)_{Q}\right](\tau)
$$

Next we consider asymptotic (or quantum) dimensions of irreducible modules

Theorem 8.1. $\quad$ (i) For $\beta$ as in Theorem 6.2, we have

$$
q \operatorname{dim}\left(W^{0}(p, \beta)_{Q}\right)=\operatorname{dim}_{\mathbb{C}} V(-\sqrt{p} \bar{\lambda}) .
$$

(ii) For any typical module $F_{\lambda}$, we have

$$
\operatorname{qdim}\left(F_{\lambda}\right)=p^{\left|\Delta_{+}\right|}
$$

Proof: It follows directly from Theorem 6.2 and the definition of analytic $q$-dimension

$$
\operatorname{qdim}[M]=\lim _{t \rightarrow 0} \frac{\operatorname{ch}[M](i t)}{\operatorname{ch}[V](i t)},
$$

where $M$ is a module for the vertex operator algebra $V$. 
Observe that $\operatorname{dim}_{\mathbb{C}}\left(V((p-1) \rho)=p^{\left|\Delta_{+}\right|}\right.$. On the other hand, because of $-\bar{\lambda}=\left(s_{1}-1\right) \omega_{1}+\cdots+$ $\left(s_{n}-1\right) \omega_{n}$, where $s_{j} \in\{1, \ldots, p\}$, we always have

$$
\operatorname{dim}_{\mathbb{C}} V(-\sqrt{p} \bar{\lambda}) \leq p^{\Delta_{+}} .
$$

Therefore we conclude that typical modules have the largest quantum dimension among irreducible modules (see also [10]).

Remark 8. Certain regularized characters of $W^{0}(p)_{Q^{-}}$-modules including their regularized quantum dimensions are studied in a recent paper [12] (see also [11]).

\section{APPENDIX A. HARMONICITY OF CERTAIN POLYNOMIALS}

In this part we prove harmonicity of certain polynomials needed for the proof of modularity of characters in Theorem 4.1.

A.1. Some harmonic polynomials of type $A$. Consider the rank $n$ root lattice of type $A$, $Q=\oplus_{j=1}^{n} \mathbb{Z} \alpha_{j}$, whose Cartan matrix $A_{n}=\left(\left\langle\alpha_{j}, \alpha_{k}\right\rangle\right)_{j, k=1}^{n}$ is given by

$$
A_{n}:=\left(\begin{array}{cccccc}
2 & -1 & 0 & \ldots & 0 & 0 \\
-1 & 2 & -1 & \ldots & 0 & 0 \\
0 & -1 & 2 & \ldots & 0 & 0 \\
\ldots & \ldots & \ldots & \ldots & 2 & -1 \\
0 & 0 & 0 & \ldots & -1 & 2
\end{array}\right) .
$$

Then $A_{n}^{-1}$ is the Cartan matrix of the dual (or weight) lattice $P=\oplus_{j=1}^{n} \mathbb{Z} \omega_{j}$ such that $\left\langle\omega_{j}, \alpha_{k}\right\rangle=\delta_{j, k}$. Clearly, $A_{n}^{-1}:=\left(a^{j k}\right)$, where $a^{j k}:=\left\langle\omega_{j}, \omega_{k}\right\rangle$.

Denote by $\nabla^{2}$ the standard (diagonal) Laplacian on $\mathbb{R}^{n}$ and let $\nabla_{B}^{2}$ be the Laplace-Beltrami operator for $B \in \operatorname{Mat}(n, \mathbb{R})$, that is

$$
\nabla_{B}^{2}:=\left(\frac{\partial}{\partial x}\right)^{T} B \frac{\partial}{\partial x}=\sum_{j, k=1}^{n} b_{j k} \partial_{j k}^{2} ; \quad \partial_{j k}^{2}:=\frac{\partial^{2}}{\partial x_{j} \partial x_{k}} .
$$

Definition 1. We say that polynomial $f\left(x_{1}, \ldots, x_{n}\right)$ is harmonic with respect to $B$ (resp. harmonic) if $\nabla_{B}^{2}(f)=0$ (resp. $\left.\nabla^{2}(f)=0\right)$.

Remark 9. Note that $f$ is harmonic with respect to $B$ if and only if it is harmonic with respect to $\lambda B$ for some $\lambda \in \mathbb{R}^{\times}$.

The following result follows easily, using basic facts from Calculus and Linear Algebra.

Lemma A.1. Consider the linear change of variable $x=B y$, where $B$ is an invertible matrix, $C \in \operatorname{Mat}(n, \mathbb{R}), x=\left(x_{1}, \ldots, x_{n}\right)$, and $y=\left(y_{1}, \ldots, y_{n}\right)$. Then $\nabla_{C}^{2}(f(x))=\nabla_{\left(B^{-1}\right)^{T} B^{-1} C}^{2}\left(f^{*}(y)\right)$, where $f^{*}(y)=f(B(y))$. In particular, $\nabla^{2}(f(x))=\nabla_{\left(B^{-1}\right)^{T} B^{-1}}^{2}\left(f^{*}(y)\right)$.

The next result is well-known (see [27, Theorem 5.1.8]).

Proposition A.2. The discriminant $\delta\left(x_{1}, \ldots, x_{n}\right):=\prod_{j<k}\left(x_{j}-x_{k}\right)$ is harmonic.

Lemma A.3. Suppose that $t \in \mathbb{R}$ and let $f\left(x_{1}, \ldots, x_{n}\right)$ be harmonic with respect to $B$. Then

$$
f\left(x_{1}+a_{1} t,, \ldots, x_{j}+a_{j} t, \ldots, x_{n}+a_{n} t\right)
$$

is also harmonic with respect to $B$ for all $a_{j} \in \mathbb{R}$. In particular,

$$
\prod_{j<k}\left(x_{j}-x_{k}+t(j-k)\right)
$$

is harmonic. 
Proof: By using Taylor's Theorem, we see that

$$
\exp \left(\sum_{j=1}^{n} t a_{j} \partial_{x_{j}}\right) f\left(x_{1}, \ldots, x_{n}\right)=f\left(x_{1}+a_{1} t, \ldots, x_{j}+a_{j} t, \ldots, x_{n}+a_{n} t\right) .
$$

The proof now follows by observing that any pair of partial differential operators with constant coefficients commute with each other, in particular

$$
\exp \left(\sum_{i=1}^{n} t a_{j} \partial_{x_{j}}\right) \circ \nabla^{2}=\nabla^{2} \circ \exp \left(\sum_{j=1}^{n} t a_{j} \partial_{x_{j}}\right) .
$$

Recall the set of positive roots of $Q$ :

$$
\Delta^{+}=\left\{\alpha_{j}, j=1, \ldots, n\right\} \cup\left\{\alpha_{j}+\cdots+\alpha_{k}: 1 \leq j<k \leq n\right\} \quad \text { and } \quad \rho=\sum_{j=1}^{n} \omega_{j} .
$$

Next we introduce several families of polynomial with respect to two bases of $\mathbb{R}^{n}$. For $\lambda=$ $x_{1} \alpha_{1}+\cdots+x_{n} \alpha_{n}$, we let

$$
\delta_{Q}\left(x_{1}, \ldots, x_{n}\right):=\prod_{\alpha \in \Delta^{+}}(\lambda+\rho, \alpha), \quad \widetilde{\delta}_{Q}\left(x_{1}, \ldots, x_{n}\right):=\prod_{\alpha \in \Delta^{+}}(\lambda, \alpha) .
$$

Similarly, for $\mu=x_{1} \omega_{1}+\cdots x_{n} \omega_{n}$, we set

$$
\delta_{P}\left(x_{1}, \ldots, x_{n}\right):=\prod_{\alpha \in \Delta^{+}}(\mu+\rho, \alpha), \quad \widetilde{\delta}_{P}\left(x_{1}, \ldots, x_{n}\right):=\prod_{\alpha \in \Delta^{+}}(\mu, \alpha) .
$$

The subscript $Q$ (resp. $P$ ) indicates that we use the $\alpha$-basis (resp. $\omega$-basis) in the paramatrization of $\lambda$ (resp. $\mu$ ).

Example 3. With $Q=A_{2}$, we have

$$
\begin{aligned}
& \delta_{Q}\left(x_{1}, x_{2}\right)=\left(2 x_{1}-x_{2}+1\right)\left(2 x_{2}-x_{1}+1\right)\left(x_{1}+x_{2}+2\right), \\
& \widetilde{\delta}_{Q}\left(x_{1}, x_{2}\right)=\left(2 x_{1}-x_{2}\right)\left(2 x_{2}-x_{1}\right)\left(x_{1}+x_{2}\right), \\
& \delta_{P}\left(x_{1}, x_{2}\right)=\left(x_{1}+1\right)\left(x_{2}+1\right)\left(x_{1}+x_{2}+2\right), \\
& \widetilde{\delta}_{P}\left(x_{1}, x_{2}\right)=x_{1} x_{2}\left(x_{1}+x_{2}\right) .
\end{aligned}
$$

From $\mu=x_{1} \omega_{1}+\cdots+x_{n} \omega_{n}$, we have

$$
\widetilde{\delta}_{P}\left(x_{1}, x_{2}, \ldots, x_{n}\right)=\prod_{k=1}^{n} x_{k} \prod_{1 \leq j<\ell \leq n}\left(x_{j}+\cdots x_{\ell}\right) .
$$

Similarly,

$$
\delta_{P}\left(x_{1}, x_{2}, \ldots, x_{n}\right)=\prod_{k=1}^{n}\left(x_{k}+1\right) \prod_{1 \leq j<\ell \leq n}\left(x_{j}+\cdots+x_{\ell}+\ell-j+1\right) .
$$

with $A_{n}$ as in (A.1), we have

Theorem A.4. The functions $\widetilde{\delta}_{P}$ and $\delta_{P}$ are harmonic with respect to $A_{n}$. The functions $\widetilde{\delta}_{Q}$ and $\delta_{Q}$ are harmonic with respect to $A_{n}^{-1}$. 
Proof: We apply Proposition A.2 for the discriminant with $n+1$ variables:

$$
\nabla^{2}\left(\delta\left(x_{1}, \ldots, x_{n+1}\right)\right)=\sum_{j=1}^{n+1} \partial_{j j}^{2} \delta\left(x_{1}, \ldots, x_{n+1}\right)=0 .
$$

We then make a change of variables:

$$
y_{1}:=x_{1}-x_{2}, \ldots, y_{n}:=x_{n}-x_{n+1}, \quad y_{n+1}:=x_{n+1} .
$$

With respect to new variables, $\delta$ does not depend on $y_{n+1}$ and

$$
\delta^{*}\left(y_{1}, \ldots, y_{n+1}\right)=\prod_{j=1}^{n} y_{\ell} \prod_{1 \leq j<k \leq n}\left(y_{j}+\cdots+y_{k}\right)=\widetilde{\delta}_{P}\left(y_{1}, \ldots, y_{n}\right) .
$$

The transition between the $y$ and $x$ variables is controlled by the $(n+1) \times(n+1)$ matrix

$$
A=\left(\begin{array}{ccccc}
1 & 0 & 0 & \ldots & 0 \\
-1 & 1 & 0 & \ldots & 0 \\
0 & -1 & 1 & \ldots & 0 \\
\ldots & \ldots & \ldots & \ldots & \ldots \\
0 & 0 & \ldots & -1 & 1
\end{array}\right)
$$

Note that we have

$$
A^{T} A=\left(\begin{array}{ccccc}
2 & -1 & 0 & \ldots & 0 \\
-1 & 2 & -1 & \ldots & 0 \\
0 & -1 & 2 & \ldots & \ldots \\
\ldots & \ldots & \ldots & \ldots & \ldots \\
0 & 0 & \ldots & \ldots & 1
\end{array}\right)
$$

Moreover observe that the principal $n \times n$ minor of $A^{T} A$ is precisely the Cartan matrix of type $A_{n}$. By using Lemma A.1, with $B^{-1}=A$, we get

$$
0=\nabla^{2}(\delta(\mathbf{x}))=\nabla_{A^{T} A}^{2}\left(\delta^{*}(\mathbf{y})\right) .
$$

Although $A^{T} A$ matrix is of size $n+1, \delta^{*}(\mathbf{y})$ does not involve the $y_{n+1}$ variable, thus only the summation over the principal minor of $A_{n}$ is relevant for the laplacian. Consequently,

$$
\nabla_{A_{n}}^{2}\left(\widetilde{\delta}_{P}(\mathbf{y})\right)=0
$$

as desired. The $A_{n}$-harmonicity of $\delta_{P}$ now easily follows from Lemma A.3

Next we consider $\delta_{Q}(\mathbf{x})$ and $\tilde{\delta}_{Q}(\mathbf{x})$. Observe that they are related to $\delta_{P}(\mathbf{x})$ and $\widetilde{\delta}_{P}(\mathbf{x})$, respectively, under a change of basis. More precisely, if we let $\mathbf{y}=A_{n} \mathbf{x}$ then

$$
\widetilde{\delta}_{P}(\mathbf{x})=\widetilde{\delta}_{Q}(\mathbf{y})
$$

Clearly, $A_{n}=A_{n}^{T}$, so $\left(A_{n}^{-1}\right)^{T}=A_{n}^{-1}$. Another application of Lemma A.1 results in

$$
0=\nabla_{A_{n}}^{2}\left(\widetilde{\delta}_{P}\right)=\nabla_{A_{n}^{-1} A_{n}^{-1} A_{n}}^{2}\left(\widetilde{\delta}_{Q}\right)=\nabla_{A_{n}^{-1}}^{2}\left(\widetilde{\delta}_{Q}\right)
$$

as desired. 
A.2. Some harmonic polynomials of $D$-type. To parametrize the roots of $D_{n}$, it is convenient to use the orthogonal basis $\varepsilon_{j}, j=1, \ldots, n$. In this basis positive roots are given by

$$
\Delta^{+}=\left\{\varepsilon_{j}-\varepsilon_{k}: 1 \leq j<k \leq n\right\} \cup\left\{\varepsilon_{j}+\varepsilon_{k}: j<k\right\} .
$$

Simple roots are $\alpha_{j}=\varepsilon_{j}-\varepsilon_{j+1}, j=1, \ldots, n-1$ and $\alpha_{n}=\varepsilon_{n-1}+\varepsilon_{n}$. With this ordering the Cartan matrix is

$$
D_{n}=\left(\begin{array}{ccccccc}
2 & -1 & 0 & \ldots & 0 & 0 & 0 \\
-1 & 2 & -1 & \ldots & 0 & 0 & 0 \\
0 & -1 & 2 & \ldots & 0 & 0 & 0 \\
\ldots & \ldots & \ldots & \ldots & \ldots & \ldots & \ldots \\
0 & 0 & 0 & \ldots & 2 & -1 & -1 \\
0 & 0 & 0 & \ldots & -1 & 2 & 0 \\
0 & 0 & 0 & \ldots & -1 & 0 & 2
\end{array}\right) .
$$

The next result is taken from [27, Section 5.1.3].

Proposition A.5. The function

$$
\prod_{j<k}\left(x_{j}^{2}-x_{k}^{2}\right)
$$

is harmonic with respect to $D_{n}$.

As before we consider

$$
\widetilde{\delta}_{Q}:=\prod_{\alpha \in \Delta^{+}}\langle\lambda, \alpha\rangle, \quad \delta_{Q}=\prod_{\alpha \in \Delta^{+}}\langle\lambda+\rho, \alpha\rangle
$$

Similarly, we define $\widetilde{\delta}_{P}$ and $\delta_{P}$.

Example 4. Let $Q=D_{4}$, then

$$
\begin{aligned}
\delta_{Q}= & \left(2 x_{1}-x_{2}\right)\left(2 x_{2}-x_{1}-x_{3}-x_{4}\right)\left(2 x_{3}-x_{2}\right)\left(2 x_{4}-x_{2}\right)\left(x_{1}+x_{2}-x_{3}-x_{4}\right)\left(x_{2}+x_{3}-x_{4}-x_{1}\right) \\
& \times\left(x_{1}+x_{3}-x_{4}\right) x_{2}\left(x_{1}+x_{3}+x_{4}-x_{2}\right)\left(x_{1}+x_{4}-x_{3}\right)\left(x_{3}+x_{4}-x_{1}\right)\left(x_{2}+x_{4}-x_{1}-x_{3}\right) .
\end{aligned}
$$

Our next result is analogous to Theorem A.4, now for the $D$-type.

Theorem A.6. The function $\widetilde{\delta}_{Q}$ is harmonic with respect to $D_{n}^{-1}$ and $\widetilde{\delta}_{P}$ is harmonic with respect to $D_{n}$.

Proof: For the proof, we use Proposition A.5 and the change-of-variables matrix

$$
D=\left(\begin{array}{ccccc}
1 & 0 & 0 & \ldots & 0 \\
-1 & 1 & 0 & \ldots & 0 \\
0 & -1 & 1 & \ldots & 0 \\
\ldots & \ldots & \ldots & \ldots & \ldots \\
0 & 0 & \ldots & -1 & 1 \\
0 & 0 & \ldots & 1 & 1
\end{array}\right)
$$

We then easily see that

$$
D^{T} D=D_{n}
$$

Together with Lemma A.1 this yields that $\widetilde{\delta}_{P}$ is harmonic with respect to $D_{n}$. Another change of variables (as in Theorem A.4) easily implies that $\widetilde{\delta}_{Q}$ is harmonic with respect to $D_{n}^{-1}$. 


\section{A.3. Towards harmonic polynomials of type $E$.}

Remark 10. For $Q=E_{6}, E_{7}$ and $E_{8}$, we have to consider,

$$
\begin{aligned}
& p_{6}\left(x_{1}, \ldots, x_{6}\right)=\nabla_{E_{6}^{-1}}^{2} \delta_{Q}\left(x_{1}, \ldots, x_{6}\right), \\
& p_{7}\left(x_{1}, \ldots, x_{7}\right)=\nabla_{E_{7}^{-1}}^{2} \delta_{Q}\left(x_{1}, \ldots, x_{7}\right), \\
& p_{8}\left(x_{1}, \ldots, x_{8}\right)=\nabla_{E_{8}^{-1}}^{2} \delta_{Q}\left(x_{1}, \ldots, x_{8}\right) .
\end{aligned}
$$

By using MAPLE, we computed $p_{j}$, for $j=6,7,8$. We were able to show that $p_{6}=p_{7}=0$. But we could not simplify these polynomials to the standard form due to a very high degree for $E_{8}$. Even so, we were able to evaluate $p_{8}$ for many randomly chosen $x_{j}$-values and all these calculations returned zero. Thus, we can conclude with a high certainty that $p_{8}$ is indeed zero.

\section{REFERENCES}

[1] D. Adamović and A. Milas, Logarithmic intertwining operators and $W(2,2 p-1)$-algebras, J. Math. Phys., 48 (2007), p. 073503.

[2] D. Adamović and A. Milas, On the triplet vertex algebra W(p), Adv. Math. 217 (2008), 2664-2699.

[3] D. Adamovic and A. Milas, An analogue of modular BPZ equation in logarithmic (super) conformal field theory, Contemporary Mathematics 497 (2009), 1-17.

[4] D. Adamović and A. Milas, $C_{2}$-cofinite $\mathcal{W}$-algebras and their logarithmic modules, to appear in the proceedings of the conference "Conformal Field Theories and Tensor Categories", Beijing, June 2011, Springer 2014.

[5] D. Adamović, X. Lin, and A. Milas, $A D E$ subalgebras of the triplet vertex algebra $\mathcal{W}(p): A$-series, Commun. Contemp. Math. 15 (2013), 1350028.

[6] G. Andrews and B. Berndt, Ramanujan's Lost Notebook: Vol.2., Springer, 2009.

[7] T. Arakawa, Representation Theory of W-Algebras, Inventiones Math. 169 (2007), 219-320.

[8] R. Borcherds, Vertex algebras, Kac-Moody algebras, and the Monster, Proc. Natl. Acad. Sci. USA 83 (1986), 3068-3071.

[9] K. Bringmann, T. Creutzig, and L. Rolen, Negative index Jacobi forms and quantum modular forms, Research in Mathematical Sciences 1 (2014), 1-32.

[10] K. Bringmann and A.Milas, W-algebras, false theta functions and quantum modular forms, I.M.R.N. 21 (2015), 11351-11387.

[11] T. Creutzig and A. Milas, The false theta functions and the Verlinde formula, Adv. Math. 262 (2014), 520-545; arXiv:1309.6037.

[12] T. Creutzig and A. Milas, Higher rank partial and false theta functions and representation theory, submitted; arXiv: 1607.08563.

[13] T. Creutzig, A.Milas, and M. Rupert, Logarithmic Link Invariants of $\bar{U}_{q}^{H}\left(\mathfrak{s l}_{2}\right)$ and Asymptotic Dimensions of Singlet Vertex Algebras, submitted; arXiv:1605.05634.

[14] T. Creutzig, A.Milas, and S. Wood, On regularized quantum dimension of the singlet vertex algebras and false theta functions, I.M.R.N (2016), rnw037; arXiv:1411.3282.

[15] A. De Sole and V. Kac, Finite vs affine W-algebras, Jap. Journal of Math. 1 (2006), 137-261.

[16] M. Eichler and D. Zagier, The Theory of Jacobi Forms Progress in Math. 55, Birkhauser-Verlag, Basel-Boston (1985), 148 pages

[17] P. Etingof, A uniform proof of the Macdonald-Mehta-Opdam identity for finite Coxeter groups, Math. Res. Lett. 17 (2010), 275-282.

[18] B. Feigin and I. Tipunin, W-algebras connected with simple Lie algebras, preprint; arXiv:1002.5047.

[19] B. Feigin and A.M. Gănutdinov, A. M. Semikhatov, and I. Yu Tipunin, I, The Kazhdan-Lusztig correspondence for the representation category of the triplet $W$-algebra in logarithmic conformal field theories. (Russian) Teoret. Mat. Fiz. 148 (2006), no. 3, 398-427.

[20] B. Feigin, A. Gaŭnutdinov, A. Semikhatov, and I. Tipunin, Logarithmic extensions of minimal models: characters and modular transformations. Nuclear Phys. B 757 (2006), 303-343.

[21] A. Folsom, K. Ono, and R. Rhoades, Mock theta functions and quantum modular forms, Forum of Mathematics, П 1 (2013) e2, 27 pages.

[22] E. Frenkel and D. Ben-Zvi, Vertex algebras and algebraic curves, Mathematical Surveys and Monographs, 88, American Mathematical Society, Providence, RI, 2001.

[23] I. Frenkel, J. Lepowsky, and A. Meurman, Vertex Operator Algebras and the Monster, Pure and Applied Math., Vol. 134, Academic Press, 1988. 
[24] J. Fuchs, S. Hwang, A. Semikhatov, and I. Tipunin, Nonsemisimple Fusion Algebras and the Verlinde Formula, Comm. Math. Phys. 247 (2004), no. 3, 713-742.

[25] S. Garoufalidis and T. Vuong, A stability conjecture for the colored Jones polynomial, preprint 2013.

[26] T. Gannon, Lattices and Theta Functions, PhD Thesis, McGill,1991.

[27] R. Goodman and N. Wallach, Symmetry, Representations and Invariants, Vol. 66. Dordrecht: Springer, 2009.

[28] R. Lawrence and D. Zagier, Modular forms and quantum invariants of 3-manifolds. Asian Journal of Mathematics 3 (1999), 93-108.

[29] J. Lepowsky and H. Li, Introduction to Vertex Operator Algebras and Their Representations, Birkhauser, Boston, 2003.

[30] A.Milas, Characters of modules of irrational vertex algebras, Contributions in Mathematical and Computational Sciences (Proceedings of the Conference on Vertex Algebras and Automorphic Forms), Heidelberg, 2011, 8 (2014), 1-29.

[31] A.Milas and M. Penn, Lattice vertex algebras and combinatorial bases: general case and $\mathcal{W}$-algebras, New York Journal of Mathematics 18, (2012) 621-650.

[32] A. Semikhatov, Virasoro central charges for Nichols algebras, Mathematical Lectures from Peking University Bai, C.; Fuchs, J.; Huang, Y.-Z.; Kong, L.; Runkel, I.; Schweigert, C. (Eds.) 2014, IX 67-92.

[33] M.-F. Vignéras, Séries thêta des formes quadratiques indéfinies, Modular Functions of One Variable VI, Springer Berlin Heidelberg, 1977, 227-239.

[34] O. Warnaar, Partial theta functions. I. Beyond the last notebook. Proc. London Math. Soc. 87 (2003), 363-395.

[35] D. Zagier, Valeurs des fonctions zêta des corps quadratiques réels aux entiers négatifs, Journées Arithmetiques de Caen 1976, Astérisque 41-42 (1977), 135-151.

[36] D. Zagier, Vassiliev invariants and a strange identity related to the Dedekind eta-function, Topology 40 (2001), 945-960.

[37] D. Zagier, Quantum modular forms, In Quanta of Maths: Conference in honor of Alain Connes, Clay Mathematics Proceedings 11, AMS and Clay Mathematics Institute 2010, 659-675.

Mathematical Institute, University of Cologne, Weyertal 86-90, 50931 Cologne, Germany

E-mail address: kbringma@math.uni-koeln.de

Department of Mathematics and Statistics, SUnY-Albany, 1400 Washington Avenue, Albany, Ny 12222, USA

E-mail address: amilas@albany.edu 